Weronika Buczyńska · Jarosław A. Wiśniewski

\title{
On geometry of binary symmetric models of phylogenetic trees
}

\author{
Dedicated to Andrzej Biatynicki-Birula
}

Received January 14, 2006, and in revised form April 24, 2006

\begin{abstract}
We investigate projective varieties which are binary symmetric models of trivalent phylogenetic trees. We prove that they have Gorenstein terminal singularities and are Fano varieties of index 4 and dimension equal to the number of edges of the tree in question. Moreover any two such varieties which are of the same dimension are deformation equivalent, that is, they are in the same connected component of the Hilbert scheme of the projective space. As an application we provide a simple formula for computing their Hilbert-Ehrhart polynomial.
\end{abstract}

Keywords. Phylogenetic tree, binary symmetric model, toric variety, Fano variety, Ehrhart polynomial, Hilbert scheme

\section{Introduction}

The grand idea of phylogenetics is to reconstruct the historical relations between species by analyzing their present features and putting their common ancestors in a diagram which forms a tree. This leads to describing the evolution in terms of a Markov process on a tree. Subsequently, by looking at the distribution of random variables associated to this process one arrives at geometric models of phylogenetic trees, which eventually yields algebraic varieties. These, in turn, can be studied in purely algebro-geometric way in order to get algebraic relations which describe the dependency between the variables encoded in the underlying tree.

Our original task was to compute the Hilbert-Ehrhart polynomial for a variety arising as a binary symmetric model of trivalent phylogenetic trees. The Hilbert-Ehrhart polynomial provides information on the number of algebraic relations defining the variety in question. Unexpectedly, however, the polynomial does not depend on the shape of the tree but merely on its size, the number of leaves or, equivalently, the dimension of its geometric model. Looking for an explanation of this phenomenon we found one of the main results of the present paper,2.24, which asserts that models of trees with the same number of leaves are deformation equivalent, that is, they are in the same connected component of the Hilbert scheme of the projective space in question (hence they have the same Hilbert polynomial).

W. Buczyńska, J. A. Wiśniewski: Instytut Matematyki Uniwersytetu Warszawskiego, Banacha 2, 02-097 Warszawa, Poland; e-mail: wkrych@ mimuw.edu.pl, J.Wisniewski@ mimuw.edu.pl

Mathematics Subject Classification (2000): Primary 14M25; Secondary 14C05, 14J45, 92B10 
The fact that the geometric models of trees modeling some processes-which are discrete objects-live in a connected continuous family of geometric objects probably deserves an explanation in terms of algebraic statistics or even biology (cf. [BHV]). For the algebraic geometry part we have a natural question of irreducibility of the component of the Hilbert scheme containing these models and (if the irreducibility is confirmed) about varieties which arise as general deformations (that is, over a general point of the component of the Hilbert scheme in question). The question about a general deformation of the model is related to the other main result of the present paper, 2.15, which is that these models are index 4 Fano varieties with Gorenstein terminal singularities. Thus one would expect that their general deformation is a smooth Fano variety of index 4 (cf. $[\mathrm{Na}]$ ).

The present paper is organized as follows. We deal with varieties defined over the complex numbers. In the first section we recall and restate definitions and results about binary symmetric models of trivalent trees. We do it on the level of toric varieties, by defining toric data associated to a tree and then using a standard toric construction of associating a variety to a polytope in a lattice. For an explanation regarding the reduction to toric varieties we refer the reader to $[\overline{\mathrm{StSu}}]$ and [ERSS], with original derivations in [EiSt] and [SSE]; another view is presented in [BuWi].

In Section 1 we also present important technical results: a fiber product formula for polytopes of trees, 1.13 and its counterpart for varieties, a quotient formula 1.20 which is a special case of 1.19 . The latter asserts that, under suitable assumptions, the toric variety associated to a fiber product of two polytopes is a Mumford's GIT (Geometric Invariant Theory) quotient of the product of the corresponding varieties. We note that 1.13 can be derived from [StSu].

The second section of the present paper contains its main results. After a brief discussion of equations defining a geometric model of a tree, with special consideration of a tree with two inner nodes and four leaves, we examine fans of geometric models and resolution of their singularities, with 2.15 being the main structural result of this part. Next we consider deformations of models of trees. The approach is, roughly, as follows: we know how to deform equations of a small tree with four leaves and one inner edge, the result of the deformation is another tree with the inner edge "mutated":<smiles>[Z]C(I)C([Hg])C(C)I</smiles>

Applying the GIT quotient formula, 1.19, we are able to use this elementary deformation associated to four-leaf trees to get a similar deformation for every inner edge of any tree, 2.22 This implies a result about deforming one geometric model to another, 2.24 .

In the last part of Section 2, we discuss the Hilbert-Ehrhart polynomial of binary symmetric models of trivalent trees. We define a relative version of the polynomial and then a product of such polynomials which is related to gluing the corresponding trees. The deformation procedure implies associativity of the product which not only implies the invariance of the Hilbert-Ehrhart polynomial for trees with the same number of leaves but also provides a simple formula for computing it, 2.36 
In the appendix we prove that a fiber product of two polytopes admitting unimodular covers has this property as well, A.3. This provides a straightforward proof of normality of polytopes associated to trivalent trees and, what is equally important, it is used in constructing corresponding normal objects in the proofs of 1.19 and 2.24 . We note that the part regarding normality of polytopes associated to trees follows from known results. Namely, in [StSu] it is proved that the ideal of the binary symmetric model of a trivalent tree has a Gröbner basis consisting of quadrics, hence by [St2, Prop. 13.15] the toric variety in question and its underlying polytope are normal.

In the last part of the appendix, using polymake] software, we verify a simple (yet 9-dimensional) example to check that the polytope models of different trees in this case are different. The question if the models of non-isomorphic trees are non-isomorphic is open (cf. [AlRh]).

The paper uses consistently the language of algebraic geometry, including toric geometry. We ignore relations to algebraic statistics and biology, suggesting the reader to look into [ $\mathrm{PaSt}],[\mathrm{SeSt}]$ or $[\mathrm{Fe}]$ (or into [ERSS] for a concise exposition), to get an idea about the background of the problems that we deal with. It was our primary intention to make the paper self-contained so that it can be read by an algebraic geometer with no knowledge of its possible applications outside algebraic geometry. On the other hand, a reader who is not familiar with algebraic geometry but is interested in acquiring ideas which are important in our approach (regarding quotients and deformations) is advised to look into $[\overline{\mathrm{Re}}]$ and $[\mathrm{Alt}]$ for a short exposition of these matters.

\subsection{Notation}

- $|\mathcal{A}|$ denotes the cardinality of a finite set $\mathcal{A}$.

- A lattice is a finitely generated free abelian group.

- Depending on the context a subscript denotes the extension of the basic ring or a fiber of a morphism, e.g. $M_{\mathbb{R}}=M \otimes_{\mathbb{Z}} \mathbb{R}$.

- Given a finite-dimensional vector space (or a lattice) $V$ with a basis $\left\{v_{1}, \ldots, v_{n}\right\}$, we will denote by $\left\{v_{1}^{*}, \ldots, v_{n}^{*}\right\}$ the dual basis of $V^{*}$, that is, $v_{i}^{*}\left(v_{i}\right)=1$ and $v_{i}^{*}\left(v_{j}\right)=0$ if $i \neq j$.

- For a vector space $V$ we denote by $\operatorname{Symm}(V)$ its symmetric algebra.

\section{Preliminaries: trees and toric geometry}

\subsection{Trees, lattices, polytopes}

Notation 1.1. A tree $\mathcal{T}$ is a simply connected graph (1-dimensional CW complex) with a set $\mathcal{E}=\mathcal{E}(\mathcal{T})$ of edges and $\mathcal{V}=\mathcal{V}(\mathcal{T})$ of vertices and the (unordered) boundary map $\partial: \mathcal{E} \rightarrow \mathcal{V}^{\wedge 2}$, where $\mathcal{V}^{\wedge 2}$ denotes the set of unordered pairs of distinct elements in $\mathcal{V}$. We note that $|\mathcal{V}|=|\mathcal{E}|+1$ where $|\mathcal{E}|$ is assumed to be positive. We write $\partial(e)=\left\{\partial_{1}(e), \partial_{2}(e)\right\}$ and say $v$ is a vertex of $e$ or $e$ contains $v$ if $v \in\left\{\partial_{1}(e), \partial_{2}(e)\right\}$; we then simply write $v \in e$. The valency of a vertex $v$ is the number of edges which contain $v$ (it is positive since $\mathcal{T}$ is 
connected and assumed to have at least one edge). A vertex $v$ is called a leaf if its valency is 1 , otherwise it is called an inner vertex or a node. If the valency of each inner node is $m$ then the tree will be called $m$-valent. The sets of leaves and nodes will be denoted $\mathcal{L}$ and $\mathcal{N}$, respectively, so $\mathcal{V}=\mathcal{L} \cup \mathcal{N}$. An edge which contains a leaf is called a petiole, an edge which is not a petiole is called an inner edge (or branch), and the set of inner edges will be denoted by $\mathcal{E}^{o}$.

Example 1.2. A caterpillar of length $n$ is a 3-valent tree with $n$ inner edges and $n+1$ inner nodes which after removing all leaves and petioles becomes just a string of edges. That is, there are exactly two inner nodes to which there are attached two petioles, and any other inner node has exactly one petiole attached.

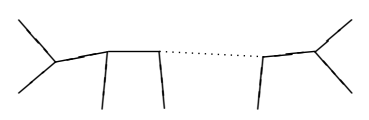

Given a tree $\mathcal{T}$ we encode it in terms of dual lattices.

Definition 1.3. Let $\mathcal{T}$ be a tree with the set $\mathcal{V}$ of vertices and $\mathcal{E}$ of edges. We define $M=M(\mathcal{T})=\bigoplus_{e \in \mathcal{E}} \mathbb{Z} \cdot e$ to be the lattice (free abelian group) spanned on the set $\mathcal{E}$. Let $N=N(\mathcal{T})=\operatorname{Hom}(M, \mathbb{Z})$ be the dual lattice. We represent elements of $\mathcal{V}$ as elements of $N$. Namely, for $v \in \mathcal{V}$ we set $v(e)=1$ if e contains the vertex $v$ and $v(e)=0$ otherwise. The pair $(M, N)$ together with the choice of the basis $\mathcal{E}$ of $M$ and the set $\mathcal{V} \subset N$ is called the lattice pair of the tree $\mathcal{T}$.

From this point on we identify the edges and vertices of $\mathcal{T}$ with the appropriate elements in $M(\mathcal{T})$ and $N(\mathcal{T})$. The elements of the basis of $N$ dual to $\{e \in \mathcal{E}\}$ will be denoted by $e^{*}$. Then for any $v \in \mathcal{V}$ we have, by definition, $v=\sum_{e \ni v} e^{*}: M \rightarrow \mathbb{Z}$. In particular, $v$ is a leaf if and only if $v=e^{*}$ for some $e$ which is a petiole for $v$.

Let us recall that $|\mathcal{V}|=|\mathcal{E}|+1$ so the set of vertices has to be linearly dependent in $N$. The set of vertices of $\mathcal{T}$ can be divided into two disjoint classes, say $\mathcal{V}=\mathcal{V}^{-} \cup \mathcal{V}^{+}$, each class consisting of vertices which can be reached from one another by passing through an even number of edges. The following observation is known.

Lemma 1.4. The equality $\sum_{v \in \mathcal{V}^{-}} v=\sum_{v \in \mathcal{V}^{+}} v$ is, up to multiplication by a constant, the only linear relation in $N$ between vectors from $\mathcal{V}$. In particular, any proper subset of $\mathcal{V}$ consists of linearly independent vectors in $N$.

Definition 1.5. Given a tree $\mathcal{T}$ with the lattice pair $(M, N)=(M(\mathcal{T}), N(\mathcal{T}))$ we define its normalized lattice pair $(\widehat{M}, \widehat{N})=(\widehat{M}(\mathcal{T}), \widehat{N}(\mathcal{T}))$ as follows: $\widehat{M}=\{u \in M: \forall v \in \mathcal{V}$ $v(u) \in 2 \mathbb{Z}\}$ and $\widehat{N}$ is a dual of $\widehat{M}$ which contains $N$ and the set $\mathcal{N} / 2=\{v / 2: v \in \mathcal{N}\}$.

Let $\square_{M}=\left\{u \in M_{\mathbb{R}}: \forall e \in \mathcal{E} 0 \leq e^{*}(u) \leq 1\right\}$ be the unit cube in the space $M_{\mathbb{R}}$. If $\Delta$ is a polytope in $M_{\mathbb{R}}$ whose vertices are contained in the set of vertices of $\square_{M}$ then we call it a $0 / 1$ polytope (or a subcube).

Definition 1.6. Given a binary tree $\mathcal{T}$ with its lattice pair $(M, N)$, its polytope model $\Delta(\mathcal{T})$ is a polytope in the lattice $M$ which is the convex hull of $\left\{u=\sum a_{i} e_{i} \in M: a_{i}=\right.$ $0,1$ and $v(u) \in 2 \mathbb{Z}$ for every $v \in \mathcal{N}\}$. 
We note that the vertices of $\Delta$ are precisely those vertices of $\square_{M}$ which are in the sublattice $\widehat{M} \subset M$. The vertices of $\square_{M}$ can be interpreted as remainders of dividing elements of $M$ by 2 or, in other words, as vectors in the linear space $M_{\mathbb{Z}_{2}}=M \otimes_{\mathbb{Z}} \mathbb{Z}_{2}$. Thus we get the following.

Lemma 1.7. If the vertices of the cube $\square_{M}$ are identified with vectors in the linear space $M \otimes \mathbb{Z}_{2}$ then the vertices of $\Delta(\mathcal{T})$ form the linear subspace $\mathcal{N}^{\perp} \subset M \otimes \mathbb{Z}_{2}$ of zeros of the forms $v \in N \otimes \mathbb{Z}_{2}$, where $v \in \mathcal{N}(\mathcal{T})$.

Corollary 1.8. The polytope $\Delta(\mathcal{T})$ has $2^{|\mathcal{L}|-1}$ vertices.

Proof. We use 1.7: by 1.4 the $v$ 's in $\mathcal{N}$ are linearly independent in $N \otimes \mathbb{Z}_{2}$ so the dimension of the space of their zeros in $M \otimes \mathbb{Z}_{2}$ is $|\mathcal{E}|-|\mathcal{N}|=|\mathcal{L}|-1$.

Example 1.9. An $m$-star tree is a tree with one inner node and $m \geq 3$ leaves. The 3-star tree will be denoted by $\lambda$. The vertices of the polytope $\Delta$ (人) with edges $e_{0}, e_{1}, e_{2}$ are as follows: $0, e_{1}+e_{2}, e_{2}+e_{0}$ and $e_{0}+e_{1}$ so that $\Delta$ (人) is a 3-dimensional tetrahedron. If $\widehat{M} \subset M$ is the sublattice spanned by the vertices of $\Delta$ (人) then $M / \widehat{M} \cong \mathbb{Z}_{2}$.

We note that the inequalities defining $\Delta$ (人) are as follows:

$(-v / 2)(\cdot) \geq-1, \quad\left(v / 2-e_{0}^{*}\right)(\cdot) \geq 0, \quad\left(v / 2-e_{1}^{*}\right)(\cdot) \geq 0, \quad\left(v / 2-e_{2}^{*}\right)(\cdot) \geq 0$, where (recall) $v=e_{0}^{*}+e_{1}^{*}+e_{2}^{*}$.

Construction 1.10. A pointed tree $(\mathcal{T}, \ell)$ is a pair consisting of a tree $\mathcal{T}$ and a leaf $\ell \in$ $\mathcal{L}(\mathcal{T})$. Given two pointed trees $\left(\mathcal{T}_{1}, \ell_{1}\right)$ and $\left(\mathcal{T}_{2}, \ell_{2}\right)$ we define their graft as follows: $\mathcal{T}=\mathcal{T}_{1} \ell_{1} \vee_{\ell_{2}} \mathcal{T}_{2}$ is a tree obtained by removing from each $\mathcal{T}_{i}$ the leaf $\ell_{i}$ and identifying their respective petioles, which become an inner edge of the resulting tree $\mathcal{T}$.

For example, a graft of two 3-star trees with distinguished leaves denoted by $\circ$ is the following operation:<smiles>CC(C)OC(C)CC(C)C(C)C</smiles>

We note that any 3-valent tree is obtained from 3-star trees by a sequence of consecutive grafts.

Let us take two lattices $M_{i}, i=1,2$, with distinguished bases $\left\{e_{0}^{i}, \ldots, e_{m_{1}}^{i}\right\}$ and corresponding $0 / 1$ polytopes $\Delta_{i}$, with the sets of vertices $\mathcal{A}_{i}$. Let $\ell_{i}=\left(e_{0}^{i}\right)^{*}: M_{i} \rightarrow \mathbb{Z}$ be the projection to the zeroth coordinate; by abuse of notation, $\ell_{i}$ will also denote its composition with the projection $M_{1} \times M_{2} \rightarrow M_{i} \rightarrow \mathbb{Z}$. Now we can take the fiber product of these objects: $M_{1} \ell_{1} \times \ell_{2} M_{2} \subset M_{1} \times M_{2}$ consists of the pairs $\left(u_{1}, u_{2}\right)$ such that $\ell_{1}\left(u_{1}\right)=\ell_{2}\left(u_{2}\right)$. In other words, $M_{1} \ell_{1} \times \ell_{2} M_{2}=\operatorname{ker}\left(\ell_{1}-\ell_{2}\right)$ and $\Delta_{1} \ell_{1} \times \ell_{2} \Delta_{2}=$ $\left(\Delta_{1} \times \Delta_{2}\right) \cap \operatorname{ker}\left(\ell_{1}-\ell_{2}\right)$.

The proof of the following observation is fairly standard and we skip it.

Lemma 1.11. In the above situation $\Delta=\Delta_{1 \ell_{1}} \times_{\ell_{2}} \Delta_{2}$ is a $0 / 1$ polytope in $M=M_{1 \ell_{1}} \times \ell_{2}$ $M_{2}$ with the set of vertices $\mathcal{A}=\mathcal{A}_{1 \ell_{1} \times \ell_{2}} \mathcal{A}_{2}$. In general, if $\Delta_{i} \subset\left(M_{i}\right)_{\mathbb{R}}$ and $\ell_{i}: M_{i} \rightarrow \mathbb{Z}$ are lattice homomorphisms such that $\ell_{i}\left(\Delta_{i}\right) \subset[0,1]$ then the set of vertices of $\Delta=$ $\Delta_{1} \ell_{1} \times \ell_{2} \Delta_{2}$ is the fiber product of the vertices of $\Delta_{i}$ 's. 
Example 1.12. Let us consider two copies of a tetrahedron, as in Example 1.9. That is, for $i=1,2$, in a lattice $M^{i}=\mathbb{Z} e_{0}^{i} \oplus \mathbb{Z} e_{1}^{i} \oplus \mathbb{Z} e_{2}^{i}$, we consider the tetrahedron $\Delta_{i}^{3}$ spanned on the vertices $0, e_{0}^{i}+e_{1}^{i}, e_{1}^{i}+e_{2}^{i}$ and $e_{2}^{i}+e_{0}^{i}$. We take the projections $\left(e_{0}^{i}\right)^{*}: M^{i} \rightarrow \mathbb{Z}$ and in the fiber product

$$
M=M_{1} \ell_{1} \times \ell_{2} M_{2}=\operatorname{ker}\left(\left(e_{0}^{2}-e_{0}^{1}\right)^{*}\right) \subset M_{1} \times M_{2}
$$

we denote by $e_{0}$ the element $e_{0}^{1}+e_{0}^{2}$. The resulting fiber product of tetrahedrons

$$
\Delta=\left(\Delta_{1}^{3} \times \Delta_{2}^{3}\right) \cap M_{\mathbb{R}}
$$

has the following vertices: $0, e_{1}^{1}+e_{2}^{1}, e_{1}^{2}+e_{2}^{2}, e_{1}^{1}+e_{2}^{1}+e_{1}^{2}+e_{2}^{2}, e_{0}+e_{1}^{1}+e_{1}^{2}, e_{0}+e_{1}^{1}$ $+e_{2}^{2}, e_{0}+e_{2}^{1}+e_{1}^{2}, e_{0}+e_{2}^{1}+e_{2}^{2}$.

Proposition 1.13. Let $\left(\mathcal{T}_{1}, \ell_{1}\right)$ and $\left(\mathcal{T}_{2}, \ell_{2}\right)$ be two pointed trees. Then

$$
\begin{aligned}
& \widehat{M}\left(\mathcal{T}_{1 \ell_{1}} \vee_{\ell_{2}} \mathcal{T}_{2}\right)=\widehat{M}\left(\mathcal{T}_{1}\right)_{\ell_{1}} \times \ell_{2} \widehat{M}\left(\mathcal{T}_{2}\right), \\
& \Delta\left(\mathcal{T}_{1} \ell_{1} \vee_{\ell_{2}} \mathcal{T}_{2}\right)=\Delta\left(\mathcal{T}_{1}\right)_{\ell_{1}} \times \ell_{2} \Delta\left(\mathcal{T}_{2}\right) .
\end{aligned}
$$

Proof. Let $M_{1}=M\left(\mathcal{T}_{1}\right), M_{2}=M\left(\mathcal{T}_{2}\right)$, and similarly for $N$ 's, $\widehat{M}$ ' and $\widehat{N}$ 's. We set $\mathcal{T}=\mathcal{T}_{1} \ell_{1} \vee_{\ell_{2}} \mathcal{T}_{2}$. Then, by Construction $1.10, M=M(\mathcal{T})=M_{1} \ell_{1} \times \ell_{2} M_{2}$ and $\square_{M}=$ $\square_{M_{1} \ell_{1} \times \ell_{2}} \square_{M_{2}}$. The two projections $p_{i}: M \rightarrow M_{i}$ yield the corresponding injections of $\operatorname{Hom}(\cdot, \mathbb{Z})$-spaces, $\iota_{i}: N_{i} \hookrightarrow N$ (in fact $\left.N=\left(N_{1} \times N_{2}\right) / \mathbb{Z}\left(\ell_{1}-\ell_{2}\right)\right)$. If $\mathcal{N}_{i}$ and $\mathcal{N}$ denote, respectively, the inner nodes of $\mathcal{T}_{i}$ and $\mathcal{T}$ then $\mathcal{N}=\iota_{1}\left(\mathcal{N}_{1}\right) \cup \iota_{2}\left(\mathcal{N}_{2}\right)$. Since $\widehat{N}, \widehat{N}_{1}, \widehat{N}_{2}$ are defined by extending $N, N_{1}, N_{2}$ by $\mathcal{N} / 2, \mathcal{N}_{1} / 2$ and $\mathcal{N}_{2} / 2$, respectively, it follows that $\widehat{N}=\widehat{N}_{1}+\widehat{N}_{2}$ in $N_{\mathbb{R}}$. This implies the first equality of the lemma. Similarly, since the set $\mathcal{N}$ determines those vertices of $\nabla_{M}$ which span $\Delta(\mathcal{T})$ we get the second equality.

Thus, in view of the above result, the structure of the polytope $\Delta(\mathcal{T})$ can be described briefly as follows: $\Delta(\mathcal{T})$ is the fiber product of the polytopes of the star trees associated to the inner nodes of the tree, fibered over the relations encoded in the inner branches of the tree. Since $\Delta(\lambda)$ is a 3-dimensional tetrahedron, this is especially straightforward in the case of 3-valent trees because $\Delta(\mathcal{T})$ is then the product of copies of the tetrahedron labeled by the inner nodes of $\mathcal{T}$, fibered by the relations presented by the inner branches of $\mathcal{T}$.

\subsection{Toric variety arising from a tree}

First, we recall the construction of a projective toric variety from a lattice polytope of characters. For generalities regarding toric geometry we refer the reader to [Fu $]$ and [Oda $]$. Let $\widehat{M}$ and $\widehat{N}$ be dual lattices of characters and 1-parameter subgroups for an algebraic torus $T_{\widehat{N}}=\widehat{N} \otimes_{\mathbb{Z}} \mathbb{C}^{*}$.

Definition 1.14. A lattice polytope $\Delta \subset \widehat{M}_{\mathbb{R}}$ is called normal if

- the sublattice of $\widehat{M}$ spanned by the differences of points in $\Delta \cap \widehat{M}$ is equal to $\widehat{M}$,

- for every integer $d \geq 0$ any point in $d \Delta \cap \widehat{M}$ is equal to a sum of $d$ points in $\Delta \cap \widehat{M}$. 
Equivalently, the second condition in the above definition can be restated as follows. Let $\widehat{M}^{\prime}=\widehat{M} \oplus \mathbb{Z}$ and take an affine map $i_{1}: \widehat{M} \rightarrow \widehat{M}^{\prime}$ such that $i_{1}(u)=(u, 1)$. Then $\Delta$ is normal in $\widehat{M}$ if and only if the semigroup spanned in $\widehat{M}^{\prime}$ by $i_{1}(\Delta \cap \widehat{M})$ is equal to the semigroup of lattice points in the cone spanned in $\widehat{M}_{\mathbb{R}}^{\prime}$ by $i_{1}(\Delta)$, that is, the semigroup $\mathbb{R}_{\geq 0}\left(i_{1}(\Delta)\right) \cap \widehat{M}^{\prime}$.

Definition 1.15. Suppose that $\Delta$ is a normal polytope in $\widehat{M}$. Let $A_{\Delta}^{d}$ be a $\mathbb{C}$-linear space with the basis $\left\{\chi^{u}: u \in d \Delta \cap \widehat{M}\right\}$. We consider the graded $\mathbb{C}$-algebra $A(\Delta)=\bigoplus_{d \geq 0} A_{\Delta}^{d}$ with multiplication $\chi^{u_{1}} \chi^{u_{2}}=\chi^{u_{1}+u_{2}}$. Then $X(\Delta)=\operatorname{Proj} A(\Delta)$ is called the projective model of $\Delta$.

We note that in the above situation $A(\Delta)$ is a normal ring, that is, it is integrally closed in its field of fractions. This, by definition, is equivalent to saying that the affine spectrum $\operatorname{Spec}(A(\Delta))$ is a normal affine variety. In fact, in such a case $A(\Delta)$ is the semigroup algebra of $\mathbb{R}_{\geq 0}\left(i_{1}(\Delta)\right) \cap \widehat{M}^{\prime}$ so $\operatorname{Spec}(A(\Delta))$ is an affine toric variety with the big torus $T_{\widehat{N} \oplus \mathbb{Z}}$. In the projective case we have the following general result which summarizes the properties of the projective model of a normal polytope (see [Oda, Sect. 2.1-2.4], [St1], $[$ St2, Sect. 13] or $[\overline{\mathrm{Fu}}])$. We denote $\operatorname{Proj}\left(\operatorname{Symm}\left(A_{\Delta}^{1}\right)\right) \cong \mathbb{P}^{|\Delta \cap \widehat{M}|-1}$ by $\mathbb{P}_{\Delta}$.

Proposition 1.16. Suppose that $\Delta$ is a normal polytope in the lattice $\widehat{M}$ of characters of a torus $T_{\widehat{N}}$. Then the following holds:

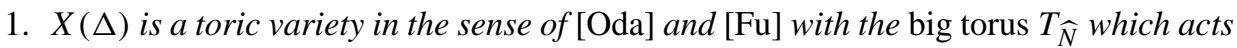
effectively on $X(\Delta)$ with an open orbit.

2. $X(\Delta)$ is embedded in $\mathbb{P}_{\Delta}$ as a projectively normal variety such that $H^{0}\left(X(\Delta), \mathcal{O}_{X}(d)\right)$ $=A_{\Delta}^{d}$.

3. The characters from $\Delta \cap \widehat{M}$ define a diagonal action of $T_{\widehat{N}}$ on $\mathbb{P}_{\Delta}$ which restricts to the torus action on $X(\Delta)$ so that the inclusion $X(\Delta) \hookrightarrow \mathbb{P}_{\Delta}$ is $T_{\widehat{N}}$-equivariant.

4. The induced action of $T_{\widehat{M}}$ on $H^{0}\left(X(\Delta), \mathcal{O}_{X}(d)\right)$ is linearizable with weights in $d \Delta \cap \widehat{M}$.

5. $X(\Delta) \subset \mathbb{P}_{\Delta}$ is the closure of the image of the map $T_{\widehat{M}} \rightarrow \mathbb{P}_{\Delta}$ defined by the characters from $\Delta \cap \widehat{M}$.

Because of A.5 the polytope model $\Delta(\mathcal{T})$ of a 3-valent tree $\mathcal{T}$ is normal so we can consider its projective model as defined above.

Definition 1.17. Let $\mathcal{T}$ be a 3-valent tree. Then the variety $X(\mathcal{T}):=X(\Delta(\mathcal{T}))$ in the projective space $\mathbb{P}_{\mathcal{T}}:=\mathbb{P}_{\Delta(\mathcal{T})}$ is called the binary symmetric model of the tree $\mathcal{T}$.

The above toric model of a phylogenetic tree is, in fact, obtained from a binary symmetric model in algebraic statistics via Fourier transform (or diagonalization). This reduction is explained in [StSu$]$ and [ERSS], with original derivations in $[\mathrm{EvSp}]$ and [SeSt $]$. Another purely algebro-geometric view is provided in [BuWi].

It is worth noting that the fiber product formula 1.13 as well as normality of $\Delta(\mathcal{T})$ (see A.5) are also consequences of results of [StSu]. More generally, using the language of $[\mathrm{StSu}]$, its main theorem implies that group based models with friendly labeling functions yield polytopes that are fiber products. 


\subsection{1-parameter group action, quotients}

In this section we consider quotients of projective varieties as in Mumford's GIT [Mu]. For a comprehensive exposition of the theory, including a relevant definition of good quotient we refer to $[\overline{\mathrm{B}-\mathrm{B}}]$. In the present section as well as in Section 2.3 we consider an algebraic action of the multiplicative group $\mathbb{C}^{*}$ on a projective variety $X \hookrightarrow \mathbb{P}^{m}$. The action is given by a choice of weights, hence it extends to the affine cone over $X$ which determines its linearization, its set of semi-stable points $X^{s s}$ and its good quotient $X^{s s} \rightarrow X^{s s} / / \mathbb{C}^{*}$ (see [B-B, Ch. 6]).

Construction 1.18. Let $\Delta_{i} \subset\left(\widehat{M}_{i}\right)_{\mathbb{R}}$ for $i=1,2$ be two lattice polytopes admitting unimodular covers, hence normal (see A.1), and let $X\left(\Delta_{i}\right) \subset \mathbb{P}^{n_{i}-1}$, where $n_{i}=$ $\left|\widehat{M}_{i} \cap \Delta_{i}\right|$, be their associated toric varieties. In $M^{\times}=\widehat{M}_{1} \times \widehat{M}_{2}$ we take the product polytope $\Delta^{\times}=\Delta_{1} \times \Delta_{2}$ which is also normal because of A.2. Then the associated toric variety $X^{\times}=X\left(\Delta^{\times}\right) \subset \mathbb{P}^{n_{1} n_{2}-1}$ is the Segre image of $X\left(\Delta_{1}\right) \times X\left(\Delta_{2}\right)$.

Suppose that $\ell_{i}: \widehat{M}_{i} \rightarrow \mathbb{Z}$ are lattice homomorphisms such that $\left(\ell_{i}\right)_{\mathbb{R}}\left(\Delta_{i}\right) \subset[0,1]$. We lift $\ell_{i}$ to the product of the lattices and on $\widehat{M}_{1} \times \widehat{M}_{2}$ we define the form $\ell_{1}-\ell_{2}$. It defines a diagonal action $\lambda_{\ell_{1}-\ell_{2}}$ of $\mathbb{C}^{*}$ on $X^{\times} \subset \mathbb{P}^{n_{1} n_{2}-1}$, which on the coordinate associated to $\chi^{\left(u_{1}, u_{2}\right)}$, where $u_{i} \in \Delta_{i} \cap \widehat{M}_{i}$, has the weight $\ell_{1}\left(u_{1}\right)-\ell_{2}\left(u_{2}\right) \in\{-1,0,1\}$. Accordingly, we regroup the coordinates of $\mathbb{P}^{n_{1} n_{2}-1}$ and write them as $\left[z_{i}^{-}, z_{j}^{0}, z_{k}^{+}\right]$depending on whether they are of weight $-1,0$ and 1 , respectively. That is,

$$
\lambda_{\ell_{1}-\ell_{2}}(t)\left[z_{i}^{-}, z_{j}^{0}, z_{k}^{+}\right]=\left[t^{-1} z_{i}^{-}, z_{j}^{0}, t z_{k}^{+}\right] .
$$

The above formula defines an action of $\lambda_{\ell_{1}-\ell_{2}}$ on the cone over $X^{\times}$and thus a $\mathbb{C}^{*}$ linearization of the bundle $\mathcal{O}_{X^{\times}}(1)$ in the sense of GIT. Denote by $X^{0}$ the intersection of $X^{\times}$with the complement of the space spanned on the eigenvectors of $\lambda_{\ell_{1}-\ell_{2}}$ of weight $\neq 0$, that is, $X^{0}=X^{\times} \backslash\left\{\left[z_{i}^{-}, z_{j}^{0}, z_{k}^{+}\right]: \forall j z_{j}^{0}=0\right\}$.

We set $\widehat{M}=\operatorname{ker}\left(\ell_{1}-\ell_{2}\right)$ and $\Delta=\Delta^{\times} \cap \operatorname{ker}\left(\ell_{1}-\ell_{2}\right)=\Delta_{1} \ell_{1} \times \ell_{2} \Delta_{2}$. By A.4 the polytope $\Delta$ is normal and thus we denote by $X(\Delta)$ its associated toric variety.

Proposition 1.19. In the above situation $X^{0}$ is equal to the set of semistable points of the action of $\lambda_{\ell_{1}-\ell_{2}}$. The projection to the weight 0 eigenspace, $\left[z_{i}^{-}, z_{j}^{0}, z_{k}^{+}\right] \mapsto\left[z_{j}^{0}\right]$, defines a regular map of $X^{0}$ to $X(\Delta)$, and $X(\Delta)$ is a good quotient for the action of $\lambda_{\ell_{1}}-\ell_{2}$.

Proof. The sections of $\mathcal{O}_{X^{\times}}(m)$ for $m>0$ make up a vector space spanned on $\chi^{u}$, where $u \in m \Delta^{\times} \cap M^{\times}$. Among them, those which are invariant with respect to the action of $\lambda_{\ell_{1}-\ell_{2}}$ are associated to $u$ 's in the intersection with $\operatorname{ker}\left(\ell_{1}-\ell_{2}\right)$, thus in $m \Delta \cap M$. By the normality of $\Delta$ (see A.4), the algebra of invariant sections is generated by those from $\mathcal{O}_{X^{\times}}(1)$. Thus the set of semistable points of the action of $\lambda_{\ell_{1}-\ell_{2}}$ is where at least one of the coordinates $z_{j}^{0}$ is non-zero and the quotient map is the projection to the weight zero eigenspace.

Corollary 1.20. Let $\left(\mathcal{T}_{1}, \ell_{1}\right)$ and $\left(\mathcal{T}_{2}, \ell_{2}\right)$ be two pointed trees. Then $X\left(\mathcal{T}_{1} \ell_{1} \vee_{\ell_{2}} \mathcal{T}_{2}\right)$ is a good quotient of $X\left(\mathcal{T}_{1}\right) \times X\left(\mathcal{T}_{2}\right)$ with respect to an action of $\lambda_{\ell_{1}-\ell_{2}}$. 
Example 1.21. Consider the $\mathbb{C}^{*}$-action on the product $\mathbb{P}_{1}^{3} \times \mathbb{P}_{2}^{3}$ given by the formula

$$
\lambda(t)\left(\left[z_{0}^{1}, z_{1}^{1}, z_{2}^{1}, z_{3}^{1}\right],\left[z_{0}^{2}, z_{1}^{2}, z_{2}^{2}, z_{3}^{2}\right]\right)=\left(\left[z_{0}^{1}, t z_{1}^{1}, t z_{2}^{1}, z_{3}^{1}\right],\left[z_{0}^{2}, t^{-1} z_{1}^{2}, t^{-1} z_{2}^{2}, z_{3}^{2}\right]\right)
$$

where the superscripts of the coordinates indicate the factor in the product $\mathbb{P}_{1}^{3} \times \mathbb{P}_{2}^{3}$. The following rational map $\mathbb{P}_{1}^{3} \times \mathbb{P}_{2}^{3}-\rightarrow \mathbb{P}^{7}$ is $\lambda$-equivariant and regular outside the set $\left\{z_{0}^{1}=z_{3}^{1}=z_{1}^{2}=z_{2}^{2}=0\right\} \cup\left\{z_{1}^{1}=z_{2}^{1}=z_{0}^{2}=z_{3}^{2}=0\right\}$, each component of which is a quadric $\mathbb{P}^{1} \times \mathbb{P}^{1}$ :

$$
\left(\left[z_{0}^{1}, z_{1}^{1}, z_{2}^{1}, z_{3}^{1}\right],\left[z_{0}^{2}, z_{1}^{2}, z_{2}^{2}, z_{3}^{2}\right]\right) \mapsto\left[z_{0}^{1} z_{0}^{2}, z_{0}^{1} z_{3}^{2}, z_{1}^{1} z_{1}^{2}, z_{1}^{1} z_{2}^{2}, z_{2}^{1} z_{1}^{2}, z_{2}^{1} z_{2}^{2}, z_{3}^{1} z_{0}^{2}, z_{3}^{1} z_{3}^{2}\right] .
$$

If $\left[x_{0}, \ldots, x_{7}\right]$ are the coordinates in $\mathbb{P}^{7}$ then the image of this map is the intersection of two quadrics, $\left\{x_{0} x_{7}=x_{1} x_{6}\right\} \cap\left\{x_{2} x_{5}=x_{3} x_{4}\right\}$.

The above claim will be clear if we write the functions $z_{i}^{1} z_{j}^{2}$ in terms of characters of the relevant torus, which we denote by $e_{i}^{1}$ and $e_{j}^{2}$, respectively. Namely, dividing the right hand side of the above displayed formula by $z_{0}^{1} z_{0}^{2}$ we get the following sequence of rational functions (cf. 1.9):

$$
\begin{array}{r}
{\left[1, \chi^{e_{1}^{2}+e_{2}^{2}}, \chi^{e_{0}^{1}+e_{1}^{1}} \chi^{e_{0}^{2}+e_{1}^{2}}, \chi^{e_{0}^{1}+e_{1}^{1}} \chi^{e_{0}^{2}+e_{2}^{2}}, \chi^{e_{0}^{1}+e_{2}^{1}} \chi^{e_{0}^{2}+e_{1}^{2}},\right.} \\
\\
\left.\chi^{e_{0}^{1}+e_{2}^{1}} \chi^{e_{0}^{2}+e_{2}^{2}}, \chi^{e_{1}^{1}+e_{2}^{1}}, \chi^{e_{1}^{1}+e_{2}^{1}} \chi^{e_{1}^{2}+e_{2}^{2}}\right] .
\end{array}
$$

If we write the sums of the exponents of the above rational functions in $M_{1} \oplus M_{2}$ and set $e_{0}=e_{0}^{1}+e_{0}^{2}$ then we get the vertices of $\Delta$ (人 $\vee$ 人 $)$ which we computed in Example 1.12 Note that from the above formula one can read off the weights with which the 1-parameter groups $\lambda_{\left(e_{j}^{i}\right)^{*}}, i, j=1,2$, associated to leaves, act on the quotient variety in $\mathbb{P}^{7}$.

\section{Geometry of phylogenetic trees}

\subsection{Paths, networks and sockets}

From now on we assume that $\mathcal{T}$ is a 3-valent tree and $X(\mathcal{T})$ in the space $\mathbb{P}_{\mathcal{T}}=\mathbb{P}_{\Delta(\mathcal{T})} \cong$ $\mathbb{P}^{2^{|\mathcal{L}|-1}-1}$ is its binary symmetric model defined by the polytope $\Delta(\mathcal{T})$. The linear coordinates on the ambient projective space are identified with the vertices of $\Delta(\mathcal{T})$ which are among the vertices of the cube $\square_{M}$ satisfying the parity relation with respect to the forms $v \in \mathcal{N} \subset N$, as in 1.6 .

Definition 2.1. A path $\gamma$ of length $m \geq 1$ on a 3-valent tree $\mathcal{T}$ is a choice of $m+1$ distinct vertices $v_{0}, \ldots, v_{m}$ such that $v_{0}$ and $v_{m}$ are leaves (called the ending points of $\gamma$ ) and there exist $m$ edges, $e_{1}, \ldots, e_{m}$, such that $\partial\left(e_{i}\right)=\left\{v_{i-1}, v_{i}\right\}$ for $i=1, \ldots, m$. A network of paths (or just a network) $\Gamma$ on $\mathcal{T}$ is a set of paths (possibly empty), no two of which have a common vertex.

A tree $\mathcal{T}$ is labeled if its leaves are numbered by $1, \ldots,|\mathcal{L}|$. Any subset $\mu \subset \mathcal{L}$ is represented by its characteristic sequence $\kappa(1), \ldots, \kappa(|\mathcal{L}|)$, where $\kappa(i)=1$ or 0 depending on whether the leaf numbered $i$ is in $\mu$ or not. A socket of a labeled tree $\mathcal{T}$ is a subset of 
$\mathcal{L}$ which has an even number of elements; each socket of $\mathcal{T}$ is identified with its characteristic sequence. For any network of paths $\Gamma$ on $\mathcal{T}$ we define its socket $\mu(\Gamma) \subset \mathcal{L}$ to be the set of leaves which are ending points of paths in $\Gamma$.

Example 2.2. Let us consider a labeled 3-valent tree with four leaves. In the following diagram, in the upper row we draw all possible networks on this tree, where paths are denoted by solid line segments. In the lower row we write down the characteristic sequences of the respective sockets.

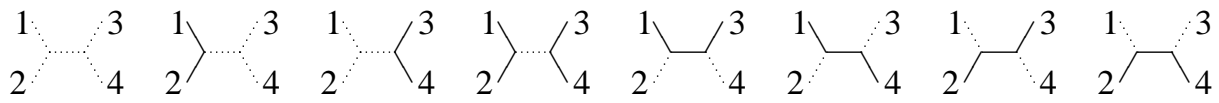

$$
\begin{aligned}
& 0,0,0,0 \quad 1,1,0,0 \quad 0,0,1,1 \quad 1,1,1,1 \quad 1,0,1,0 \quad 1,0,0,1 \quad 0,1,1,0 \quad 0,1,0,1
\end{aligned}
$$

Lemma 2.3. Let $\mathcal{T}$ be a 3-valent tree. Associating to a network $\Gamma$ the point $u(\Gamma)=$ $\sum_{e} \Gamma(e) \cdot e \in M(\mathcal{T})$, where $\Gamma(e)=1,0$ depending on whether $e$ is on $\Gamma$ or not, defines a bijection between networks and vertices of $\Delta(\mathcal{T})$.

Proof. First note that $u(\Gamma) \in \Delta(\mathcal{T})$. To define the inverse of $\Gamma \mapsto u(\Gamma)$, for any vertex $u=\sum_{e \in \mathcal{E}} \epsilon_{e} \cdot e \in \Delta(\mathcal{T})$ we define the support of $u$ to consist of the edges of $\mathcal{T}$ whose contribution to $u$ is non-zero, i.e. $\left\{e \in \mathcal{E}: e^{*}(u)=1\right\}$. The parity condition (for all $v \in \mathcal{N}$ either $v(u)=0$ or $v(u)=2$ ) implies that these edges define a network on $\mathcal{T}$.

We note that, because of 1.8 there are $2^{|\mathcal{L}|-1}$ networks. On the other hand, the association of the socket to a network gives a map from the set of networks to the subsets of leaves. This map is surjective, that is, every subset $\mu$ of $\mathcal{L}$ with an even number of elements is the socket of a network. Indeed, this follows by a straightforward induction with respect to the number of leaves of the tree: in the induction step we write a tree $\mathcal{T}_{n+1}$ with $n+1$ leaves as a graft of a tree $\mathcal{T}_{n}$ with $n$ leaves and a 3 -star tree $入$ and consider three cases depending on how many of the two new leaves replacing the single old one are in the set $\mu \subset \mathcal{L}$. Finally, because the number of all subsets of $\mathcal{L}$ with an even number of elements equals $2^{|\mathcal{L}|-1}$ we get the following.

Lemma 2.4. Let $\mathcal{T}$ be a 3-valent tree. Then associating to a network its socket defines a bijection between the set of networks of paths on $\mathcal{T}$ and the set of subsets of $\mathcal{L}$ which have an even number of elements.

Construction 2.5. Using networks and sockets, and the toric formalism, one can restate the inclusion $X(\mathcal{T}) \subset \mathbb{P}_{\mathcal{T}}$ as follows. Let $\widetilde{M}=\bigoplus_{\kappa \neq 0} \mathbb{Z} \cdot \kappa$ be the free abelian group generated by the non-empty sockets of a tree $\mathcal{T}$. We interpret the empty socket $\kappa=0$ as the zero of the lattice. Define $\mathbb{P}_{\mathcal{L}}$ as the toric variety $X\left(\widetilde{\Delta}^{0}\right)$ associated to the unit simplex $\widetilde{\Delta}^{0}$ in $\widetilde{M}$ spanned on the vectors of the distinguished basis.

Now the bijective map sockets $\rightarrow$ networks gives rise to a homomorphism of lattices $\widetilde{M} \rightarrow \widehat{M}(\mathcal{T})$, where (recall 1.5 , the latter lattice is spanned in $M(\mathcal{T})$ by the points associated to networks. This gives the identification $\mathbb{P}_{\mathcal{L}}=\mathbb{P}_{\mathcal{T}}$ and a surjective map from the symmetric graded algebra spanned by all the sockets, which is just the algebra of polynomials $\mathbb{C}\left[\chi^{\kappa}\right]$, to the algebra $A(\Delta)$, hence the inclusion $X(\mathcal{T}) \hookrightarrow \mathbb{P}_{\mathcal{L}}$ (cf. Section 1.2). 
As stated in 1.16 there is an action of the torus $T_{\widehat{N}}$ on $\mathbb{P}_{\mathcal{T}}$ which makes the embedding $X(\mathcal{T}) \hookrightarrow \mathbb{P}_{\mathcal{T}}$ equivariant. Using the identification $\mathbb{P}_{\mathcal{T}}=\mathbb{P}_{\mathcal{L}}$ we can easily describe the action of each leaf on $\mathbb{P}_{\mathcal{L}}$. Namely, the 1-parameter group $\lambda_{\ell}$ associated to a leaf $\ell$ acts on the coordinate $x_{\kappa}$, where $\kappa$ denotes the characteristic function of a socket, with weight $\kappa(\ell)$ which is one if $\ell$ is in the socket and zero otherwise. If $N_{\mathcal{L}}=\bigoplus_{v \in \mathcal{L}} \mathbb{Z} \cdot v$ is the sublattice of $N$ spanned by the leaves and $T_{N_{\mathcal{L}}}=N_{\mathcal{L}} \otimes_{\mathbb{Z}} \mathbb{C}^{*}$ its associated torus then the above rule defines an action of $T_{N_{\mathcal{L}}}$ on $\mathbb{P}_{\mathcal{L}}$ which, in fact, does not depend on the actual shape of the tree $\mathcal{T}$ but only on the labeling of the leaves.

Example 2.6. The equations defining in $\mathbb{P}_{\mathcal{T}}=\mathbb{P}_{\mathcal{L}}$ the model of a tree with four leaves from Example 1.21 can be described in terms of relations between networks representing relevant points in $\Delta(\mathcal{T})$ (cf. Example 2.2. They are as follows:

$$
\left.\frac{1}{2} \cdot \begin{array}{l}
3 \\
4
\end{array}+\frac{1}{2}\right\rangle\left\langle\begin{array}{l}
3 \\
4
\end{array}=\frac{1}{2}\right\rangle \cdot \frac{3}{4}+\frac{1}{2}\left\langle_{4}^{3}\right.
$$

and

$$
{ }_{2}^{1} \backslash_{4}^{3}+{ }_{2}^{1} r_{4}^{3}={ }_{2}^{1} \nearrow_{4}^{3}+\frac{1}{2} \nearrow_{4}^{3}
$$

In other words, using the coordinates $x_{\kappa(1) \cdots \kappa(4)}$ in $\mathbb{P}_{\mathcal{L}}$, the respective equations defining $X(\mathcal{T})$ are as follows:

$$
\begin{aligned}
& x_{0000} \cdot x_{1111}=x_{1100} \cdot x_{0011}, \\
& x_{1001} \cdot x_{0110}=x_{1010} \cdot x_{0101} .
\end{aligned}
$$

Next, note that renumbering the leaves as below or, equivalently, changing the shape of a 3-valent tree connecting the four numbered leaves, produces the following equations, respectively:

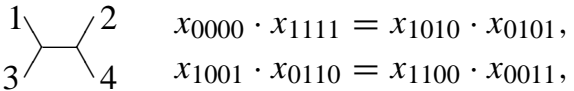

$$
\begin{aligned}
& 1)-\begin{array}{ll}
2 \quad x_{0000} \cdot x_{1111}=x_{1001} \cdot x_{0110}, \\
3 & x_{1001} \cdot x_{0110}=x_{1010} \cdot x_{0101}
\end{array}
\end{aligned}
$$

We note that all the above equations involve only four quadratic monomials: $x_{0000} x_{1111}$, $x_{1100} x_{0011}, x_{1010} x_{0101}, x_{1001} x_{0110}$. Moreover, given any leaf $\ell$, the 1-parameter group $\lambda_{\ell}$ acts with weight 1 on each of these monomials. Thus the model in $\mathbb{P}_{\mathcal{L}}$ of each of the above three trees is invariant with respect to the action of $T_{N_{\mathcal{L}}}$.

\subsection{Dual polytopes, fans, resolutions and Fano varieties}

In the situation of 1.15 the fan of the variety $X(\Delta)$ in $\widehat{N}$ can be described in terms of its support functions [Oda, Thm. 2.22] or dual polytopes [Fu]. 
Example 2.7. By looking at Example 1.9 and the inequalities which appear there we see that the fan of $X(\lambda)$ in $\widehat{N} \supset N$ has rays generated by the following elements: $-v / 2=$ $-\left(e_{0}^{*}+e_{1}^{*}+e_{2}^{*}\right) / 2, v / 2-e_{0}^{*}=\left(e_{1}^{*}+e_{2}^{*}-e_{0}^{*}\right) / 2, v / 2-e_{1}^{*}=\left(e_{0}^{*}+e_{2}^{*}-e_{1}^{*}\right) / 2$, $v / 2-e_{2}^{*}=\left(e_{0}^{*}+e_{1}^{*}-e_{2}^{*}\right) / 2$.

The formula from 1.13 can be used to describe the polytope dual to $\Delta(\mathcal{T})$, and hence to describe the fan of $X(\mathcal{T})$ for 3 -valent trees.

Lemma 2.8. Let $\mathcal{T}$ be a 3-valent tree with $n$ inner nodes. Then the polytope $\Delta(\mathcal{T})$ is defined in $M_{\mathbb{R}}$ by the following $4 n$ inequalities: for any inner node $v \in \mathcal{N}$ at which edges $e_{v, 0}, e_{v .1}, e_{v .2}$ meet, so that $v=e_{v .0}^{*}+e_{v .1}^{*}+e_{v .2}^{*}$, we take

$(-v / 2)(\cdot) \geq-1, \quad\left(v / 2-e_{v .0}^{*}\right)(\cdot) \geq 0, \quad\left(v / 2-e_{v .1}^{*}\right)(\cdot) \geq 0, \quad\left(v / 2-e_{v .2}^{*}\right)(\cdot) \geq 0$.

Proof. Let $\left(\mathcal{T}_{1}, \ell_{1}\right)$ and $\left(\mathcal{T}_{2}, \ell_{2}\right)$ be pointed trees. If $\Delta_{i}=\Delta\left(\mathcal{T}_{i}\right) \subset\left(M_{i}\right)_{\mathbb{R}}$ is defined by inequalities with respect to some forms $w_{j}^{i}$ in $\left(N_{i}\right)_{\mathbb{R}}$ then $\Delta_{1} \times \Delta_{2}$ is defined by the forms $\left(w_{j}^{1}, 0\right)$ and $\left(0, w_{j}^{2}\right)$ in $\left(N_{1}\right)_{\mathbb{R}} \times\left(N_{2}\right)_{\mathbb{R}}$. Then the classes of these forms in $N=\left(N_{1} \times N_{2}\right)_{\mathbb{R}} / \mathbb{R}\left(\ell_{1}-\ell_{2}\right)$ define the fiber product of $\Delta_{i}$ 's. Therefore the lemma follows by induction with respect to the number of inner nodes of $\mathcal{T}$.

Definition 2.9. For a binary symmetric 3-valent tree $\mathcal{T}$ we define a polytope $\Delta^{\vee}(\mathcal{T})$ in $N_{\mathbb{R}}$ which is the convex hull of $-v / 2=-\left(e_{v .0}^{*}+e_{v .1}^{*}+e_{v .2}^{*}\right) / 2, v / 2-e_{v .0}^{*}=\left(e_{v .1}^{*}+\right.$ $\left.e_{v .2}^{*}-e_{v .0}^{*}\right) / 2, v / 2-e_{v .1}^{*}=\left(e_{v .0}^{*}+e_{v .2}^{*}-e_{v .1}^{*}\right) / 2, v / 2-e_{v .2}^{*}=\left(e_{v .0}^{*}+e_{v .1}^{*}-e_{v .2}^{*}\right) / 2$, for all inner nodes $v \in \mathcal{N}$, where $e_{v .0}, e_{v .1}, e_{v .2}$ are the edges containing $v$.

Let us note that the points listed above are in fact vertices of $\Delta^{\vee}(\mathcal{T})$. Indeed, by looking at the points which $\operatorname{span} \Delta^{\vee}(\mathcal{T})$ we see that $\left(e_{v .0}+e_{v .1}+e_{v .2}\right)\left(\Delta^{\vee}(\mathcal{T})\right) \geq-3 / 2$, with equality only for the point $-\left(e_{v .0}^{*}+e_{v .1}^{*}+e_{v .2}^{*}\right) / 2$, which is therefore a vertex. Similarly, $\left(e_{v .0}+e_{v .1}-e_{v .2}\right)\left(\Delta^{\vee}(\mathcal{T})\right) \leq 3 / 2$, with equality only for $\left(e_{v .0}^{*}+e_{v .1}^{*}-e_{v .2}^{*}\right) / 2$.

Lemma 2.10. Let $\widehat{\sigma}=\sum_{e \in \mathcal{E}}$ e. Then the polytopes $4 \Delta(\mathcal{T})-2 \widehat{\sigma}$ and $\Delta^{\vee}(\mathcal{T})$ are dual, or polar, to each other in the sense that

$$
\begin{aligned}
& \Delta^{\vee}(\mathcal{T})=\left\{w \in N_{\mathbb{R}}: w(4 \Delta(\mathcal{T})-2 \widehat{\sigma}) \geq-1\right\}, \\
& 4 \Delta(\mathcal{T})-2 \widehat{\sigma}=\left\{u \in M_{\mathbb{R}}: u\left(\Delta^{\vee}(\mathcal{T})\right) \geq-1\right\} .
\end{aligned}
$$

Proof. The first equality is a restatement of 2.8, the second follows because the polar polytope of the polar is the original polytope [Fu, Sect. 1.5].

Notation 2.11. For any vertex $u$ of $\Delta(\mathcal{T})$ we define its dual face $u^{\perp}=\Delta^{\vee}(\mathcal{T}) \cap\{w$ : $w(4 u-2 \widehat{\sigma})=-1\}$. Let $\widetilde{u}^{\perp}$ be the polytope which is the convex hull of $u^{\perp}$ and $0 \in N_{\mathbb{R}}$, while $\widehat{u}^{\perp}$ will denote the cone spanned in $N_{\mathbb{R}}$ by $u^{\perp}$.

Let $u$ be a vertex of $\Delta(\mathcal{T})$ which can be represented as a network of paths, $\Gamma(u)$. Then for $v \in \mathcal{N}$ the number $v(u)$ is either 0 or 2, depending on whether or not $\Gamma(u)$ contains $v$, and similarly $e^{*}(u)$ is respectively 0 or 1 . Thus $(-v / 2)(4 u-2 \widehat{\sigma})=-1$ if $v$ is in $\Gamma(u)$, and $(-v / 2)(4 u-2 \widehat{\sigma})=3$ otherwise. On the other hand, $\left(v / 2-e_{v .0}^{*}\right)(4 u-2 \widehat{\sigma})=-1$ if 
either $v$ is not in $\Gamma(u)$, or both $v$ and $e_{v .0}^{*}$ are in $\Gamma(u)$. Finally, $\left(v / 2-e_{v .0}^{*}\right)(4 u-2 \widehat{\sigma})=3$ if $v$ is in $\Gamma(u)$ but $e_{v, 0}^{*}$ is not.

Therefore, for any vertex $u$ of $\Delta(\mathcal{T})$ and any node $v \in \mathcal{N}$ exactly three of the four points $-v / 2=-\left(e_{v .0}^{*}+e_{v .1}^{*}+e_{v .2}^{*}\right) / 2, v / 2-e_{v .0}^{*}=\left(e_{v .1}^{*}+e_{v .2}^{*}-e_{v .0}^{*}\right) / 2, v / 2-e_{v .1}^{*}=$ $\left(e_{v .0}^{*}+e_{v .2}^{*}-e_{v .1}^{*}\right) / 2, v / 2-e_{v .2}^{*}=\left(e_{v .0}^{*}+e_{v .1}^{*}-e_{v .2}^{*}\right) / 2$ are in $u^{\perp}$, which therefore has $3 n$ vertices.

Example 2.12. We will visualize the points of $\widehat{N}$ on the graph of the tree in the following way. Given a 3-valent node $v$ with edges $e_{v .0}, e_{v .1}, e_{v .2}$, which for simplicity we just mark by numbers on the graph, the point $-v / 2$ will be denoted by the dot at the vertex, while the point $v / 2-e_{v .0}^{*}$ by the secant opposite to the edge $e_{v .0}$ :

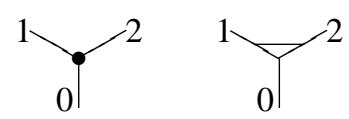

Using this notation we can put in the same picture both the network of paths associated to a vertex $u$ of $\Delta(\mathcal{T})$ and the corresponding points in $u^{\perp}$. We draw only four out of eight networks from 2.2 since the others are obtained by renumbering the leaves.
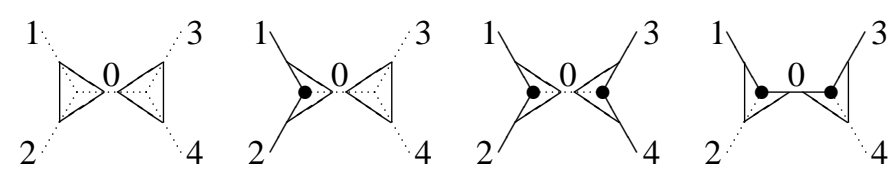

In each case the polytope $\widetilde{u}^{\perp}$ can be divided into two simplexes, each having edges which form a basis of the lattice $\widehat{N}$. For example:<smiles>CC1CC1CC1CC1COC1CC1CC1CC1</smiles>

The first equality means that $\widetilde{u}^{\perp}$ in this case is the union of the simplex with edges $\left(e_{1}^{*}+e_{2}^{*}-e_{0}^{*}\right) / 2,\left(e_{0}^{*}+e_{2}^{*}-e_{1}^{*}\right) / 2,\left(e_{0}^{*}+e_{1}^{*}-e_{2}^{*}\right) / 2,\left(e_{3}^{*}+e_{4}^{*}-e_{0}^{*}\right) / 2,\left(e_{0}^{*}+e_{3}^{*}-e_{4}^{*}\right) / 2$ and another one with edges $\left(e_{1}^{*}+e_{2}^{*}-e_{0}^{*}\right) / 2,\left(e_{0}^{*}+e_{2}^{*}-e_{1}^{*}\right) / 2,\left(e_{0}^{*}+e_{1}^{*}-e_{2}^{*}\right) / 2$, $\left(e_{3}^{*}+e_{4}^{*}-e_{0}^{*}\right) / 2,\left(e_{0}^{*}+e_{4}^{*}-e_{3}^{*}\right) / 2$. The common part of these two simplexes is the simplex with edges $\left(e_{1}^{*}+e_{2}^{*}-e_{0}^{*}\right) / 2,\left(e_{0}^{*}+e_{2}^{*}-e_{1}^{*}\right) / 2,\left(e_{0}^{*}+e_{1}^{*}-e_{2}^{*}\right) / 2,\left(e_{3}^{*}+e_{4}^{*}-e_{0}^{*}\right) / 2$, which contains $e_{0}^{*} / 2=\left(\left(e_{0}^{*}+e_{3}^{*}-e_{4}^{*}\right) / 2+\left(e_{0}^{*}+e_{4}^{*}-e_{3}^{*}\right) / 2\right) / 2$.

This example is even more transparent when we write $\widehat{N}$ as the sum of the rank 2 lattice spanned by $\left(e_{1}^{*}+e_{2}^{*}-e_{0}^{*}\right) / 2$ and $\left(e_{3}^{*}+e_{4}^{*}-e_{0}^{*}\right) / 2$, and the rank 3 lattice spanned by $\left(e_{0}^{*}+e_{2}^{*}-e_{1}^{*}\right) / 2,\left(e_{0}^{*}+e_{1}^{*}-e_{2}^{*}\right) / 2,\left(e_{0}^{*}+e_{3}^{*}-e_{4}^{*}\right) / 2$ which contains also $\left(e_{0}^{*}+e_{4}^{*}-e_{3}^{*}\right) / 2$. Then our division of the cone $\widehat{u}^{\perp}$ comes by multiplying by the cone $\mathbb{R}_{\geq 0}\left(e_{1}^{*}+e_{2}^{*}-e_{0}^{*}\right)+$ $\mathbb{R}_{\geq 0}\left(e_{3}^{*}+e_{4}^{*}-e_{0}^{*}\right)$ the standard simplicial division of the 3 -dimensional cone generated by $\left(e_{0}^{*}+e_{2}^{*}-e_{1}^{*}\right) / 2,\left(e_{0}^{*}+e_{1}^{*}-e_{2}^{*}\right) / 2,\left(e_{0}^{*}+e_{3}^{*}-e_{4}^{*}\right) / 2$ and $\left(e_{0}^{*}+e_{4}^{*}-e_{3}^{*}\right) / 2($ see $[\mathrm{Fu}$, p. 49]), which in geometric terms is a small resolution of a 3-dimensional quadric cone singularity giving rise to the so-called Atiyah flop. 
The same argument works whenever $\Gamma(u)$ does not contain $e_{0}$. Then $u^{\perp}$ contains $\left(e_{0}^{*}+e_{2}^{*}-e_{1}^{*}\right) / 2,\left(e_{0}^{*}+e_{1}^{*}-e_{2}^{*}\right) / 2,\left(e_{0}^{*}+e_{4}^{*}-e_{3}^{*}\right) / 2,\left(e_{0}^{*}+e_{3}^{*}-e_{4}^{*}\right) / 2$ and we can make a similar division of $u^{\perp}$ using the equality

$$
\left(e_{0}^{*}+e_{2}^{*}-e_{1}^{*}\right) / 2+\left(e_{0}^{*}+e_{1}^{*}-e_{2}^{*}\right) / 2=\left(e_{0}^{*}+e_{4}^{*}-e_{3}^{*}\right) / 2+\left(e_{0}^{*}+e_{3}^{*}-e_{4}^{*}\right) / 2 .
$$

If $\Gamma(u)$ contains $e_{0}$ then we use the identity

$$
-\left(e_{0}^{*}+e_{1}^{*}+e_{2}^{*}\right) / 2+\left(e_{1}^{*}+e_{2}^{*}-e_{0}^{*}\right) / 2=-\left(e_{0}^{*}+e_{3}^{*}+e_{4}^{*}\right) / 2+\left(e_{3}^{*}+e_{4}^{*}-e_{0}^{*}\right) / 2
$$

which presents $-e_{0}^{*} / 2 \in u^{\perp}$ as an average (median) of two different pairs of vertices and provides a similar decomposition

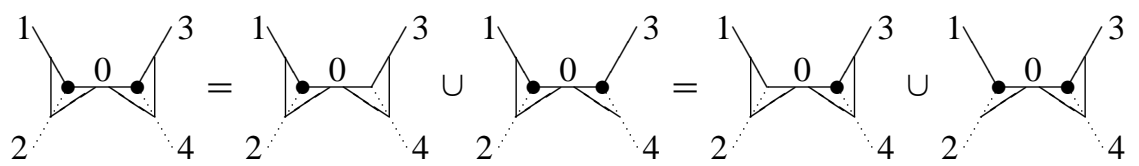

Now we shall show that the above discussion can be generalized to the case of trees with more inner nodes.

Lemma 2.13. Suppose that $\mathcal{T}$ is a binary symmetric 3-valent tree with $n$ inner nodes. Then for any vertex $u$ of $\Delta(\mathcal{T})$ there exists a division of $u^{\perp}$ (or equivalently, of $\tilde{u}^{\perp}$ ) into a union of $2^{n-1}$ (normalized) volume 1 simplexes. Equivalently, the cone $\widehat{u}^{\perp}$ can be divided into a union of simplicial cones which are regular (i.e. their generators form bases of $\widehat{N}$ ).

Proof. The construction of the division will proceed along an ascending sequence of subtrees of $\mathcal{T}$, starting from an inner node of $\mathcal{T}$. That is, we have an ascending sequence of 3-valent trees

$$
\mathcal{T}_{1} \subset \cdots \subset \mathcal{T}_{n}=\mathcal{T}
$$

where $\mathcal{T}_{i}$ has $i$ inner nodes and $\mathcal{T}_{i+1}=\mathcal{T}_{i} \vee$. Forgetting the edges which are not in $\mathcal{T}_{i}$ gives a sequence of surjective maps $M(\mathcal{T}) \rightarrow \cdots \rightarrow M\left(\mathcal{T}_{i}\right) \rightarrow \cdots \rightarrow M\left(\mathcal{T}_{1}\right)$ which yields a sequence of inclusions $\widehat{N}\left(\mathcal{T}_{1}\right) \subset \cdots \subset \widehat{N}\left(\mathcal{T}_{i}\right) \subset \cdots \subset \widehat{N}\left(\mathcal{T}_{n}\right)$. The restriction of the network $u$ to $\mathcal{T}_{i}$ is a network on $\mathcal{T}_{i}$ as well and we will denote it by $u_{i}$. Clearly $u^{\perp} \cap N\left(\mathcal{T}_{i}\right)_{\mathbb{R}}=u_{i}^{\perp}$.

Now we will define the division of $u_{i}^{\perp}$ inductively. The polytope $u_{1}^{\perp}$ is just a simplex so let us assume that $u_{i}^{\perp}=\sum \delta_{i}^{j}$ where $j=1, \ldots, 2^{i-1}$ and the normalized volume of $\delta_{i}^{j}$ with respect to the lattice $\widehat{N}\left(\mathcal{T}_{i}\right)$ is 1 . Let $v^{i}$ be an inner node of $\mathcal{T}_{i+1}$ which was a leaf of $\mathcal{T}_{i}$, let $e_{0}^{i}$ be a petiole of $\mathcal{T}_{i}$ which becomes an inner edge of $\mathcal{T}_{i+1}$, and let $e_{1}^{i}$ and $e_{2}^{i}$ be the two new petioles of $\mathcal{T}_{i+1}$ which contain $v^{i}$.

Now we argue as in 2.12 If $e_{0}^{i}$ is in $u$ then $-\left(e_{0}^{i}\right)^{*} / 2 \in u_{i}^{\perp}$ and we may assume that $e_{1}^{i}$ is in $u$ and $e_{2}^{i}$ is not. Now from any simplex $\delta_{i}^{j}$ from the original division of $u_{i}^{\perp}$ we produce two simplexes by adding a new vertex at $\left(\left(e_{0}^{i}\right)^{*}+\left(e_{2}^{i}\right)^{*}-\left(e_{1}^{i}\right)^{*}\right) / 2$ and another one at either $-\left(\left(e_{0}^{i}\right)^{*}+\left(e_{1}^{i}\right)^{*}+\left(e_{2}^{i}\right)^{*}\right) / 2$ or $\left(\left(e_{1}^{i}\right)^{*}+\left(e_{2}^{i}\right)^{*}-\left(e_{0}^{i}\right)^{*}\right) / 2$. Because

$$
-\left(\left(e_{0}^{i}\right)^{*}+\left(e_{1}^{i}\right)^{*}+\left(e_{2}^{i}\right)^{*}\right) / 2+\left(\left(e_{1}^{i}\right)^{*}+\left(e_{2}^{i}\right)^{*}-\left(e_{0}^{i}\right)^{*}\right) / 2=-\left(e_{0}^{i}\right)^{*}
$$

and $-\left(e_{0}^{i}\right)^{*} / 2 \in u_{i}^{\perp}$ this defines a good division of $u_{i+1}^{\perp}$. 
If $e_{0}^{i}$ is not in $u$ then $\left(e_{0}^{i}\right)^{*} / 2 \in u_{i}^{\perp}$ and we make a similar construction but now we have to consider two cases: either none of $e_{1}^{i}, e_{2}^{i}$ is in $u$ or both are in $u$. In either case the discussion is similar to that in 2.12

In terms of toric geometry the division process implies the following.

Corollary 2.14. The affine toric variety associated to the cone $\widehat{u}^{\perp}$ has Gorenstein terminal singularities which admit a small resolution.

Proof. The toric singularities are Cohen-Macaulay and since all the generators of the rays of $\widehat{u}^{\perp}$ lie on the hyperplane $(4 u-2 \sigma)(\cdot)=-1$ the singularities in question are Gorenstein. The division into regular simplicial cones involves adding no extra ray so the appropriate resolution is small, which also implies that the original singularity is terminal.

We note that the construction of the division certainly depends on the choice of the root of the tree, and changing the root gives a flop.

Let $\Sigma$ be a fan in $\widehat{N}_{\mathbb{R}}$ consisting of the cones $\widehat{u}^{\perp}$ for every $u$ which is a vertex of $\Delta(\mathcal{T})$, and their faces. In other words, $\Sigma$ contains the cones spanned by the proper faces of $\Delta^{\vee}(\mathcal{T})$ (including the empty face, whose cone is the zero cone). Recall that equivariant line bundles on a toric variety are described in a standard way by piecewise linear functions on its fan (see [Oda, Sect. 2.1] or [Fu, Sect. 3.4]). Setting $\Lambda_{\widehat{u}^{\perp}}=u$ we define a continuous piecewise linear function $\Lambda$ on the fan $\Sigma$ in $N_{\mathbb{R}}$ such that for every $v \in \mathcal{N}$ and $e_{v} \in \mathcal{E}$ containing $v$ we have $\Lambda(-v / 2)=-1$ and $\Lambda\left(v / 2-e_{v}^{*}\right)=0$. The sections of the bundle related to $\Lambda$ (see [Oda, Prop. 2.1] or [Fu, p. 66]) are in $\widehat{M} \cap \Delta(\mathcal{T})$. Therefore the toric variety $X(\Sigma)$ given by the fan $\Sigma$ can be identified with the original variety $X(\Delta(\mathcal{T})$ ) and the line bundle associated to $\Lambda$ is $\mathcal{O}_{X}(1)$. On the other hand, the function $4 \Lambda-2 \sigma$ assumes value -1 on the primitive vectors in rays of $\Sigma$, which allows us to identify the anti-canonical divisor of $X(\Delta)$ (see [Oda, Sect 2.1]). The result is the following.

Theorem 2.15. Let $\mathcal{T}$ be a 3-valent binary symmetric tree. Then the variety $X(\mathcal{T})$ is Gorenstein and Fano with terminal singularities. Moreover it is of index 4, that is, the canonical divisor $K_{X(\mathcal{T})}$ is linearly equivalent to $\mathcal{O}_{X(\mathcal{T})}(-4)$.

We note the following consequence of Kodaira-Kawamata-Viehweg vanishing (see e.g. [KoMo, Sect. 2.5]).

Corollary 2.16. In the above situation $H^{i}(X(\mathcal{T}), \mathcal{O}(d))=0$ for $i>0$ and $d \geq-3$. In particular for $d \geq 0$ we have $\operatorname{dim}_{\mathbb{C}} H^{0}(X(\mathcal{T}), \mathcal{O}(d))=h_{X(\mathcal{T})}(d)$, the Poincaré-Hilbert polynomial of $(X(\mathcal{T}), \mathcal{O}(1))$.

\subsection{Mutation of a tree, deformation of a model}

In Example 2.6 we noted that a 4-leaf 3-valent tree can be labeled in three non-equivalent ways. We can revert it to say that given four numbered leaves we have three 3 -valent 
labeled trees connecting these leaves. By grouping in pairs the leaves whose petioles are attached to common inner nodes we can list them as follows: $(1,2)(3,4),(1,3)(2,4)$, and $(1,4)(2,3)$.

Now, given four pointed trees $\mathcal{T}_{i}, i=1, \ldots, 4$, we can produce a tree $\mathcal{T}$ by grafting the tree $\mathcal{T}_{i}$ along the $i$-th leaf of a labeled 3-valent 4-leaf tree $\mathcal{T}_{0}$. Here are the possible configurations, $e_{0}$ denotes the inner edge of the tree $\mathcal{T}_{0}$ :
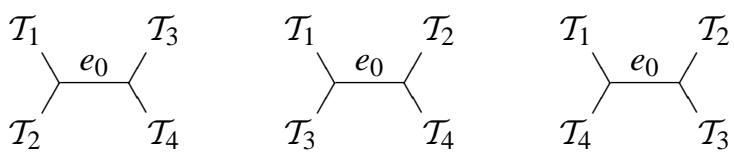

Definition 2.17. In the above situation we say that there exists an elementary mutation along $e_{0}$ from one of the above trees to the other two. (We note that a mutation may actually yield an equivalent tree.) We say that two trees are mutation equivalent if there exists a sequence of elementary mutations from one to the other.

The following observation is fairly standard and it is obtained by easy induction with respect to the number of inner nodes.

Lemma 2.18. Any two 3-valent trees with the same number of leaves are mutation equivalent.

Now, let us recall the basics regarding deforming subvarieties in projective space. Let $\mathcal{B}$ be an irreducible variety (possibly non-complete). Consider the product $\mathbb{P}^{m} \times \mathcal{B}$ with the two projections $p_{\mathbb{P}}$ and $p_{\mathcal{B}}$. Suppose that $\mathcal{X} \subset \mathbb{P}^{m} \times \mathcal{B}$ is a subscheme such that the induced projection $p_{\mathcal{B} \mid \mathcal{X}}: \mathcal{X} \rightarrow \mathcal{B}$ is proper and flat. Suppose that for two points $a, b \in \mathcal{B}$ the scheme-theoretic fibers $X_{a}=\mathcal{X}_{a}$ and $X_{b}=\mathcal{X}_{b}$ are reduced and irreducible. Then we say that the subvariety $X_{a}$ in $\mathbb{P}^{m}$ can be deformed to $X_{b}$ over the base $\mathcal{B}$. This gives rise to a notion of deformation equivalent subvarieties of $\mathbb{P}^{m}$.

Definition 2.19. Given two subvarieties $X_{1}, X_{2}$ in $\mathbb{P}^{m}$ we say that they are deformation equivalent if their classes are in the same connected component of the Hilbert scheme of $\mathbb{P}^{m}$.

Example 2.20. Let us consider $\mathbb{P}^{7}$ with homogeneous coordinates indexed by sockets of a 4-leaf tree $\mathcal{T}_{0}$, as in Example 2.6. In $\mathbb{P}^{7}$ we consider a family of intersections of two quadrics parametrized by an open subset $\mathcal{B}$ of $\mathbb{P}^{2}$ with coordinates $\left[t_{(12)(34)}, t_{(13)(24)}\right.$, $\left.t_{(14)(23)}\right]$. We set $\mathcal{B}=\mathbb{P}^{2} \backslash\left\{\left[1, \varepsilon, \varepsilon^{2}\right]: \varepsilon^{3}=1\right\}$ and over $\mathcal{B}$ we consider $\mathcal{X}^{0}$ given in $\mathcal{B} \times \mathbb{P}^{7}$ by the equations

$$
\begin{aligned}
& t_{(12)(34)} \cdot x_{1100} x_{0011}+t_{(13)(24)} \cdot x_{1010} x_{0101} \\
&+t_{(14)(23)} \cdot x_{1001} x_{0110}=\left(t_{(12)(34)}+t_{(13)(24)}+t_{(14)(23)}\right) x_{0000} x_{1111}, \\
&\left(t_{(13)(24)}-t_{(14)(23)}\right) \cdot x_{1100} x_{0011} \\
&+\left(t_{(14)(23)}-t_{(12)(34)}\right) \cdot x_{1010} x_{0101} \\
&+\left(t_{(12)(34)}-t_{(13)(24)}\right) \cdot x_{1001} x_{0110}=0 .
\end{aligned}
$$


Three special fibers of the projection $\mathcal{X}^{0} \rightarrow \mathcal{B}$, namely $\mathcal{X}_{[1,0,0]}^{0}, \mathcal{X}_{[0,1,0]}^{0}$ and $\mathcal{X}_{[0,0,1]}^{0}$, are varieties associated to three 4-leaf trees labeled by (12)(34), (13)(24) and (14)(23), respectively. On the other hand, $\mathcal{X}^{0}$ is a complete intersection of two quadrics and the map $\mathcal{X}^{0} \rightarrow \mathcal{B}$ is equidimensional. The latter statement follows because over $\mathcal{B}$ the matrix

$\left[\begin{array}{cccc}t_{(12)(34)} & t_{(13)(24)} & t_{(14)(23)} & t_{(12)(34)}+t_{(13)(24)}+t_{(14)(23)} \\ t_{(13)(24)}-t_{(14)(23)} & t_{(14)(23)}-t_{(12)(34)} & t_{(12)(34)}-t_{(13)(24)} & 0\end{array}\right]$

is of rank 2 hence any fiber over $\mathcal{B}$ is a complete intersection of two non-proportional quadrics. Hence $\mathcal{X}^{0} \rightarrow \mathcal{B}$ is flat because of [Ei, Thm. 18.16].

Denote by $T_{0}$ the 4-dimensional torus associated to the lattice spanned by the leaves with coordinates $\chi^{v_{i}^{*}}$, where $v_{i}, i=1, \ldots, 4$, are the leaves of $\mathcal{T}_{0}$. The torus $T_{0}$ acts on $\mathbb{P}^{7} \times \mathcal{B}$ via the first coordinate, that is, for a leaf $v_{i}$ of $\mathcal{T}_{0}$ and a socket $\kappa$ we have

$$
\lambda_{v_{i}}(t)\left(x_{\kappa}, t_{(\cdot)(\cdot)}\right)=\left(t^{\kappa\left(v_{i}\right)} x_{\kappa}, t_{(\cdot)(\cdot)}\right) \text {. }
$$

Then by looking at the equations defining $\mathcal{X}^{0}$ we see that the inclusion $\mathcal{X}^{0} \hookrightarrow \mathbb{P}^{7} \times \mathcal{B}$ is equivariant with respect to this action.

We also note that a rational map $\mathbb{P}^{7}-\rightarrow \mathbb{P}^{3}$, regular outside 16 linear $\mathbb{P}^{3}$, s, which is given by four quadrics:

$$
\left[x_{\kappa}\right] \mapsto\left[x_{0000} x_{1111}, x_{0011} x_{1100}, x_{0101} x_{1010}, x_{0110} x_{1001}\right]
$$

defines a good quotient with respect to the action of $T_{0}$ on $\mathbb{P}^{7}$ (cf. [B-B, 7.1.1]). If we take a subvariety $\mathcal{Z}^{0}$ in the product $\mathbb{P}^{3} \times \mathcal{B}$ defined by the equations

$$
\begin{gathered}
t_{(12)(34)} \cdot z_{1}+t_{(13)(24)} \cdot z_{2}+t_{(14)(23)} \cdot z_{3}=\left(t_{(12)(23)}+t_{(13)(24)}+t_{(14)(23)}\right) \cdot z_{0}, \\
\left(t_{(23)(14)}-t_{(14)(23)}\right) \cdot z_{1}+\left(t_{(14)(23)}-t_{(12)(34)}\right) \cdot z_{2}+\left(t_{(12)(34)}-t_{(23)(14)}\right) \cdot z_{3}=0,
\end{gathered}
$$

then $\mathcal{Z}^{0} \rightarrow \mathcal{B}$ is equidimensional and $\mathcal{X}^{0}$ is the fiber product of $\mathbb{P}^{7}-\rightarrow \mathbb{P}^{3}$ and $\mathcal{Z}^{0} \rightarrow$ $\mathbb{P}^{3}$. As a result the induced rational map $\mathcal{X}^{0}-\rightarrow \mathcal{Z}^{0}$ defines a good quotient of $\mathcal{X}^{0}$ with respect to the action of $T_{0}$ [B-B, 7.1.4].

In what follows we construct an ambient variety which contains as locally complete intersections a flat family of varieties containing a geometric model of a tree as well as models of the tree's elementary mutations.

Construction 2.21. Let $\mathcal{T}$ be a tree with an inner edge $e_{0}$ which contains two 3 -valent inner vertices. We can write $\mathcal{T}$ as a graft of five trees: a labeled tree $\mathcal{T}_{0}$ with four leaves $v_{i}$, $i=1, \ldots, 4$, containing $e_{0}$ as an inner edge and four pointed trees $\left(\mathcal{T}_{i}, \ell_{i}\right), i=1, \ldots, 4$, attached to $\mathcal{T}_{0}$ along the respectively labeled leaves. The edges in $\mathcal{T}$ which have common nodes with $e_{0}$ are denoted by $e_{i}$, where $e_{i}$ comes from a petiole of $\ell_{i}$ (or $v_{i}$ ). Recall (see 1.13 that $M(\mathcal{T})$ and $\Delta(\mathcal{T})$ can be expressed as fiber products of $M\left(\mathcal{T}_{i}\right)$ and $\Delta\left(\mathcal{T}_{i}\right)$, respectively. That is,

$$
M(\mathcal{T})=\prod_{i=0}^{4} M\left(\mathcal{T}_{i}\right) \cap \bigcap_{i=1}^{4} \operatorname{ker}\left(\ell_{i}-v_{i}\right), \quad \Delta(\mathcal{T})=\prod_{i=0}^{4} \Delta\left(\mathcal{T}_{i}\right) \cap \bigcap_{i=1}^{4} \operatorname{ker}\left(\ell_{i}-v_{i}\right) .
$$


Now, as in 2.5, we consider the lattice $\widetilde{M}_{0}$ spanned on the non-trivial sockets of the tree $\mathcal{T}_{0}$ together with the unit simplex $\widetilde{\Delta}_{0} \subset \widetilde{M}_{0} \otimes \mathbb{R}$ and the maps $\widetilde{M}_{0} \rightarrow M_{0}$ and $\widetilde{\Delta}_{0} \rightarrow \Delta_{0}$ which give the inclusion $X\left(\mathcal{T}_{0}\right) \subset \mathbb{P}^{7}$ as a complete intersection of two quadrics. The forms $v_{i}, i=1, \ldots, 4$, pull back to forms $\tilde{v}_{i}$ on $\widetilde{M}_{0}$. Now we define

$$
\begin{aligned}
& \bar{M}=\left(\tilde{M}_{0} \times \prod_{i=1}^{4} M\left(\mathcal{T}_{i}\right)\right) \cap \bigcap_{i=1}^{4} \operatorname{ker}\left(\ell_{i}-\tilde{v}_{i}\right), \\
& \bar{\Delta}=\left(\tilde{\Delta}_{0} \times \prod_{i=1}^{4} \Delta\left(\mathcal{T}_{i}\right)\right) \cap \bigcap_{i=1}^{4} \operatorname{ker}\left(\ell_{i}-\tilde{v}_{i}\right) .
\end{aligned}
$$

As in Section 1.2 we define the toric variety $\mathcal{Y}=X(\bar{\Delta})$. We note that, by A.4 the polytope $\bar{\Delta}$ is normal in the lattice $\widetilde{M}_{0} \times \prod_{i=1}^{4} \widehat{M}\left(\mathcal{T}_{i}\right) \cap \bigcap_{i=1}^{4} \operatorname{ker}\left(\ell_{i}-\tilde{v}_{i}\right)$, which is spanned by its vertices. Also, by the construction we have the embeddings $X(\mathcal{T}) \hookrightarrow \mathcal{Y} \hookrightarrow \mathbb{P}_{\mathcal{L}}$ which are induced by identifying generators of the corresponding graded algebras (cf. 2.5).

Lemma 2.22. The inclusions

$$
\bar{M} \hookrightarrow \tilde{M}_{0} \times \prod_{i=1}^{4} M\left(\mathcal{T}_{i}\right) \quad \text { and } \quad \bar{\Delta} \hookrightarrow \widetilde{\Delta}_{0} \times \prod_{i=1}^{4} \Delta\left(\mathcal{T}_{i}\right)
$$

induce a rational map

$$
\mathbb{P}^{7} \times \prod_{i=1}^{4} X\left(\mathcal{T}_{i}\right)-\rightarrow \mathcal{Y}
$$

which is a good quotient map (of the set over which it is defined) with respect to the action of the 4-dimensional torus $T_{0}$ generated by the 1-parameter groups $\lambda_{v_{i}-\ell_{i}}, i=1, \ldots, 4$. The subvariety

$$
\widehat{\mathcal{X}}=\mathcal{X}^{0} \times \prod_{i=1}^{4} X\left(\mathcal{T}_{i}\right) \hookrightarrow \mathcal{B} \times \mathbb{P}^{7} \times \prod_{i=1}^{4} X\left(\mathcal{T}_{i}\right)
$$

is $T_{0}$-equivariant and its quotient $\mathcal{X}$ is a locally complete intersection in $\mathcal{B} \times \mathcal{Y}$.

Proof. The first (quotient) part is the same as in 1.19 , this time however we repeat the argument for all four fiber products in question. The invariance of the variety $\widehat{\mathcal{X}}$ follows from the invariance of $\mathcal{X}^{0} \hookrightarrow \mathcal{B} \times \mathbb{P}^{7}$ discussed in 2.20. Finally, since $\widehat{\mathcal{X}}$ is a complete intersection in $\mathcal{B} \times \mathbb{P}^{7} \times \prod_{i=1}^{4} X\left(\mathcal{T}_{i}\right)$ its image $\mathcal{X}$ is a locally complete intersection in the quotient, which is $\mathcal{B} \times \mathcal{Y}$. This follows from the definition of good quotient which is locally an affine quotient [B-B, Ch. 5], hence functions defining $\widehat{\mathcal{X}}$ locally descend to functions defining $\mathcal{X}$.

Lemma 2.23. Over an open set $\mathcal{B}^{\prime} \subset \mathbb{P}^{2}$ containing the points $[1,0,0],[0,1,0],[0,0,1]$ the projection morphism $\mathcal{X} \rightarrow \mathcal{B}^{\prime}$ is flat. The fibers over these points are reduced and isomorphic to, respectively, the geometric model of $\mathcal{T}$ and of its elementary mutations along the edge $e_{0}$. 
Proof. First, the fibers in question, $\mathcal{X}_{[*, *, *]}$, of $\mathcal{X} \rightarrow \mathcal{B}$ are models of the claimed trees. Indeed, this follows from the universal properties of good quotients (cf. [B-B]), as they are quotients of the respective products $\mathcal{X}_{[*, *, *]}^{0} \times \prod_{i=1}^{4} X\left(\mathcal{T}_{i}\right)$, which are located, as three invariant subvarieties, in $\widehat{\mathcal{X}}=\mathcal{X}^{0} \times \prod_{i=1}^{4} X\left(\mathcal{T}_{i}\right)$. This, in particular, implies that the corresponding fibers of $\mathcal{X} \rightarrow \mathcal{B}$ are of the expected dimension, hence are contained in a set $\mathcal{B}^{\prime} \subset \mathbb{P}^{2}$ over which the map in question is equidimensional. Since $\mathcal{Y}$ is toric it is Cohen-Macaulay and because $\mathcal{X}$ is locally complete intersection in $\mathcal{Y}$, it is also CohenMacaulay [Ei, Prop. 18.13]. Finally, the map $\mathcal{X} \rightarrow \mathcal{B}^{\prime}$ is equidimensional, hence flat, because $\mathcal{B}^{\prime}$ is smooth (see [Ei, Thm. 18.16]).

Theorem 2.24. The geometric models of 3-valent trees with the same number of leaves are deformation equivalent in $\mathbb{P}_{\mathcal{L}}$.

Proof. This is a combination of 2.18 and 2.23

\subsection{Hilbert-Ehrhart polynomial}

Definition 2.25. Given two pointed trees $\left(\mathcal{T}_{1}, \ell_{1}\right)$ and $\left(\mathcal{T}_{2}, \ell_{2}\right)$ we define their pointed graft to be the pointed tree $(\mathcal{T}, o)=\left(\mathcal{T}_{1}, \ell_{1}\right) \star\left(\mathcal{T}_{2}, \ell_{2}\right)$ where $\mathcal{T}=\mathcal{T}_{1} \ell_{1} \vee_{o_{1}}$ 入 ${ }_{o_{2}} \vee_{\ell_{2}} \mathcal{T}_{2}$, and $o, o_{1}$ and $o_{2}$ are the leaves of $入$.

Example 2.26. The pointed graft of two 3 -valent stars is

$$
\rangle \circ \star 0<=\lambda_{0}
$$

By arguments used in the proof of 1.13 we also get

Proposition 2.27. Let $\left(\mathcal{T}_{1}, \ell_{1}\right)$ and $\left(\mathcal{T}_{2}, \ell_{2}\right)$ be two pointed trees. Then

$$
\Delta\left(\mathcal{T}_{1} \ell_{1} \star \ell_{2} \mathcal{T}_{2}\right)=\Delta\left(\mathcal{T}_{1}\right) \ell_{1} \times o_{1} \Delta(\lambda){ }_{o_{2}} \times \ell_{2} \Delta\left(\mathcal{T}_{2}\right)
$$

Let us consider a 3-dimensional lattice $M=\mathbb{Z} e_{0} \oplus \mathbb{Z} e_{1} \oplus \mathbb{Z} e_{2}$ with a fixed tetrahedron $\Delta^{0}$ with vertices $0, e_{0}+e_{1}, e_{0}+e_{2}, e_{1}+e_{2}$. By $\widehat{M} \subset M$ we denote the index 2 sublattice spanned on the vertices of $\Delta^{0}$.

Definition 2.28. Let $n$ be a positive integer and let $f_{1}^{n}=f_{1}, f_{2}^{n}=f_{2}$ be two functions defined on the set $\{0, \ldots, n\}$ with values in $\mathbb{Z}$ or, more generally, in an arbitrary ring or algebra (we use the superscript ${ }^{n}$ to indicate the domain of $f$ 's). For any $k \in\{0, \ldots, n\}$ we define

$$
\left(f_{1} \star f_{2}\right)(k)=\sum_{\substack{u \in \widehat{M} \cap n \Delta^{0} \\ e_{0}^{*}(u)=k}} f_{1}\left(e_{1}^{*}(u)\right) \cdot f_{2}\left(e_{2}^{*}(u)\right) .
$$

We note that $\star$ is commutative, that is, $f_{1}^{n} \star f_{2}^{n}=f_{2}^{n} \star f_{1}^{n}$, but possibly not associative. By $\left(f^{n}\right)^{\star m}$ we denote the consecutive $\star$ product of $m$ copies of $f^{n}$, that is, $f^{n} \star\left(f^{n} \star\right.$ $\left(\ldots\left(f^{n} \star f^{n}\right) \ldots\right)$. By $1^{n}$ we denote the constant function $\{0, \ldots, n\} \rightarrow\{1\} \subset \mathbb{Z}$.

A function $f^{n}:\{0, \ldots, n\} \rightarrow \mathbb{Z}$ will be called symmetric if $f^{n}(k)=f^{n}(n-k)$. 
Lemma 2.29. If $f_{1}=f_{1}^{n}, f_{2}=f_{2}^{n}:\{0, \ldots, n\} \rightarrow \mathbb{Z}$ are symmetric functions then $f_{1} \star f_{2}$ is also symmetric and for $k \leq n / 2$ we have

$$
\left(f_{1} \star f_{2}\right)(k)=2 \cdot\left(\sum_{i=0}^{k-1} \sum_{j=0}^{i} f_{1}(i) f_{2}(k+i-2 j)\right)+\left(\sum_{i=k}^{n-k} \sum_{j=0}^{k} f_{1}(i) f_{2}(k+i-2 j)\right) .
$$

In particular, for $k \leq n / 2$,

$$
\left(f_{1} \star 1\right)(k)=2 \sum_{i=0}^{k-1}(i+1) f_{1}(i)+\sum_{i=k}^{n-k}(k+1) f_{1}(i) .
$$

Proof. Let us look at sections of the tetrahedron $n \Delta^{0}$ with hyperplanes $\left(e_{0}^{*}\right)^{-1}(k)$. We picture the situation for $n=6$ and $k=0, \ldots, 6$. For every $k$ the dotted square is a suitable section of the cube $n \square_{M}$ with the lower left corner satisfying $e_{1}^{*}=e_{2}^{*}=0$. Then the section of the tetrahedron is denoted with solid lines and the points of the lattice $\widehat{M}$ inside the (closed) tetrahedron are denoted by $\bullet$.

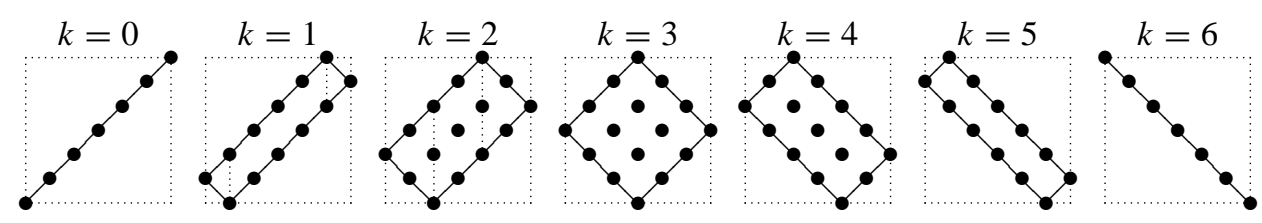

The definition of $f_{1} \star f_{2}$ involves the sum of products of $f_{i}$ 's over the lattice points of such a section. The sections over $k$ and $n-k$ are obtained by reflection with respect to either $e_{1}^{*}=n / 2$ or $e_{2}^{*}=n / 2$. Thus if one of $f_{i}$ 's is symmetric then so is $f_{1} \star f_{2}$.

On the other hand, for $0 \leq k \leq n-k$ the tetrahedron's section is a rectangle with vertices $(k, 0),(0, k),(n-k, n),(n, n-k)$ which we divide into two triangles and a parallelogram; the division is indicated by dotted vertical line segments for boxes labeled by $k=1,2$ in the above diagram. Because the functions $f_{i}$ are symmetric the values of the product $f_{1} \cdot f_{2}$ are the same for two points which are symmetric with respect to the center of the square. Thus in the formula of the lemma we take the value $f_{1}(a) f_{2}(b)$ for all integral pairs $(a, b)$ in the left hand side triangle and multiply it by 2 (that yields the first summand in the formula) and add the sum over the parallelogram.

Example 2.30. We note that $\left(1^{n}\right)^{\star 2}(k)=(k+1)(n-k+1)$ is the number of lattice points in the rectangle used in the argument in the above proof of 2.29 On the other hand, by using the formula from 2.29 one gets

$$
\left(1^{n}\right)^{\star 3}(k)=\frac{1}{6}(k+1)(n-k+1)\left(n^{2}+k n-k^{2}+5 n+6\right) .
$$

Let us recall that given a lattice polytope $\Delta \subset \widehat{M}_{\mathbb{R}}$, we define the Ehrhart function $h_{\Delta}$ as follows:

$$
h_{\Delta}(n)=|n \cdot \Delta \cap \widehat{M}| \quad \text { for any positive integer } n .
$$


If moreover $\Delta$ is normal, as in Section 1.2 then $h_{\Delta}=h_{X(\Delta)}$ where the latter is the Poincaré-Hilbert polynomial of $(X(\Delta), \mathcal{O}(1))$, which, by definition, is equal to $\operatorname{dim}_{\mathbb{C}} H^{0}(X(\Delta), \mathcal{O}(m))$ for $m \gg 0$ (cf. 1.16 ).

Definition 2.31. Let $\Delta \subset \widehat{M}_{\mathbb{R}}$ be a lattice polytope which is not contained in any hyperplane and let $v$ be a non-zero form on $\widehat{M}$. Suppose that $v(\Delta) \subset[0,1]$. We define the relative Ehrhart function $f_{\Delta, v}^{n}:\{0, \ldots, n\} \rightarrow \mathbb{Z}$ with respect to the lattice $\widehat{M}$ by setting

$$
f_{\Delta, v}^{n}(k)=\left|v^{-1}(k) \cap n \cdot \Delta \cap \widehat{M}\right|
$$

We note that, clearly, $\sum_{k=0}^{n} f_{\Delta, v}^{n}(k)=h_{\Delta}(n)$ is the usual Ehrhart function. Thus, for normal polytopes the above definition can be restated in a purely geometric fashion.

Lemma 2.32. Suppose that $\Delta$ is a normal lattice polytope and $v$ is as in 2.31. Consider a linearization of the action of the 1-parameter group $\lambda_{v}$ on $H^{0}(X(\Delta), \mathcal{O}(n))$ which has non-negative weights and the eigenspace of the zero weight is non-trivial. Then $f_{\Delta, v}^{n}(k)$ is equal to the dimension of the eigenspace of the action of $\lambda_{v}$ of weight $k$.

Proof. This is a consequence of the standard properties of $X(\Delta), 1.164$.

Lemma 2.33. Let $\left(\mathcal{T}_{1}, \ell_{1}\right),\left(\mathcal{T}_{2}, \ell_{2}\right)$ be two pointed trees and let $f_{\ell_{1}}^{n}$ and $f_{\ell_{2}}^{n}$ be the relative Ehrhart functions associated to $\Delta\left(\mathcal{T}_{1}\right), \Delta\left(\mathcal{T}_{2}\right)$, respectively. If $(\mathcal{T}, o)=\left(\mathcal{T}_{1}, \ell_{1}\right) \star$ $\left(\mathcal{T}_{2}, \ell_{2}\right)$ and $f_{o}^{n}$ is the relative Ehrhart function associated to $\Delta(\mathcal{T})$ then $f_{o}^{n}=f_{\ell_{1}}^{n} \star f_{\ell_{2}}^{n}$.

Proof. The two definitions of $\star$ are consistent.

Example 2.34. By using 2.30 we find out that

$$
\sum_{k=0}^{n}\left(1^{n}\right)^{\star 2}(k)=\frac{(n+1)(n+2)(n+3)}{6},
$$

which is the Poincaré-Hilbert polynomial of $\left(\mathbb{P}^{3}, \mathcal{O}(1)\right)$, while

$$
\sum_{k=0}^{n}\left(1^{n}\right)^{\star 3}(k)=\frac{(n+1)(n+2)(n+3)\left(n^{2}+4 n+5\right)}{30},
$$

which is the Poincaré-Hilbert polynomial of the intersection of two quadrics in $\mathbb{P}^{7}$.

Theorem 2.35. Consider three pointed trees $\left(\mathcal{T}_{i}, \ell_{i}\right), i=1,2,3$, with relative Ehrhart functions $f_{i}^{n}=f_{\ell_{i}}^{n}$ associated to the polytopes $\Delta\left(\mathcal{T}_{i}\right)$, respectively. Then

$$
\left(f_{1}^{n} \star f_{2}^{n}\right) \star f_{3}^{n}=f_{1}^{n} \star\left(f_{2}^{n} \star f_{3}^{n}\right) .
$$


Proof. Let $\ell$ denote the distinguished leaf of the result of the $\star$ operation on the trees. Then the relative Ehrhart functions $\left(f_{1}^{n} \star f_{2}^{n}\right) \star f_{3}^{n}$ and $f_{1}^{n} \star\left(f_{2}^{n} \star f_{3}^{n}\right)$ are respectively related to the following trees, each of which is obtained by an elementary mutation from the other:

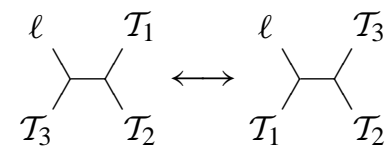

Now we repeat Construction 2.21, with obvious modifications. Namely, we define a polytope

$$
\bar{\Delta}=\left(\widetilde{\Delta}_{0} \times \prod_{i=1}^{3} \Delta\left(\mathcal{T}_{i}\right)\right) \cap \bigcap_{i=1}^{3} \operatorname{ker}\left(\ell_{i}-\tilde{v}_{i}\right)
$$

where $\widetilde{\Delta}_{0}$ is the unit simplex as in 2.5 . We define a toric variety $\mathcal{Y}=X(\bar{\Delta})$ with the embedding in $\mathbb{P}_{\mathcal{L}}$ and the action of the group $\lambda_{\ell}$.

Next, as in 2.22 we define a subvariety $\mathcal{X} \subset \mathcal{B} \times \mathcal{Y}$ such that the projection $p_{\mathcal{B}}: \mathcal{X} \rightarrow$ $\mathcal{B}$ is flat and its two fibers are varieties associated to the above two pointed trees (see 2.23). By flatness the sheaf $\left(p_{\mathcal{B}}\right)_{*}\left(p_{\mathcal{Y}}^{*}(\mathcal{O}(n))\right)$ is locally free for each $n \geq 0$ (see [Ha, III.9.9, III.12.9] and 2.16. Moreover, by construction, the action of the group $\lambda_{\ell}$ on $\mathcal{Y}$ leaves $\mathcal{X} \subset \mathcal{B} \times \mathcal{Y}$ invariant, as noted in 2.20. Finally, the decomposition into eigenspaces of the action of $\lambda_{\ell}$ on $H^{0}(\mathcal{Y}, \mathcal{O}(n))$ restricts to a corresponding eigenspace decomposition of the action of $\lambda_{\ell}$ on the fibers of $\left(p_{\mathcal{B}}\right)_{*}\left(p_{\mathcal{Y}}^{*}(\mathcal{O}(n))\right)$, which are equal to $H^{0}\left(\mathcal{X}_{b}, \mathcal{O}(n)\right)$ for $b \in \mathcal{B}$. This implies that the dimension of those eigenspaces is locally constant with respect to $b \in \mathcal{B}$, hence the relative Ehrhart function of fibers of $p_{\mathcal{B}}$ is constant, which concludes the argument.

Let us underline the fact that although the invariance of the Hilbert polynomial is a standard property of a flat family the above result is about the invariance of the family with respect to an action of a 1-parameter group, the group $\lambda_{\ell}$ in our case.

Theorem 2.35 implies that the operation $\star$ on the relative Ehrhart functions of polytopes of 3-valent trees is not only commutative (which is obvious from its definition) but also associative. This implies that the function depends neither on the shape of the trees in question nor on the location of the leaf. Therefore we have the following formula which allows one to compute the Hilbert-Ehrhart polynomial very efficiently.

Corollary 2.36. If $(\mathcal{T}, \ell)$ is a pointed 3 -valent tree with $r+1$ leaves then

$$
f_{\Delta(\mathcal{T}), \ell}^{n}=\left(1^{n}\right)^{\star r}
$$

\section{A. Appendix}

\section{A.1. Normal polytopes, unimodular covers}

A lattice simplex $\Delta^{0} \subset M_{\mathbb{R}}$ with vertices $v_{0}, \ldots, v_{r}$ is called unimodular if the vectors $v_{1}-v_{0}, \ldots, v_{r}-v_{0} \operatorname{span} M$. We say that a lattice polytope $\Delta \subset M_{\mathbb{R}}$ has a unimodular covering if $\Delta=\bigcup_{v} \Delta_{v}^{0}$ where the $\Delta_{v}^{0}$ are unimodular simplexes. This definition is taken from $[\overline{B G T}]$ where we also have the following result. 
Lemma A.1. If a lattice polytope $\Delta \subset M_{\mathbb{R}}$ has a unimodular covering then it is normal.

The following observation is probably known but we include its proof because of the proof of the subsequent lemma.

Lemma A.2. Let $\Delta_{1} \subset\left(M_{1}\right)_{\mathbb{R}}$ and $\Delta_{2} \subset\left(M_{2}\right)_{\mathbb{R}}$ be two unimodular simplexes. Then $\Delta_{1} \times \Delta_{2}$ has a unimodular covering in $M_{1} \times M_{2}$.

Proof. We can assume that the simplex $\Delta_{1}$ has vertices $0, e_{1}, \ldots, e_{r}$ and $\Delta_{2}$ has vertices $0, f_{1}, \ldots, f_{s}$. Suppose that $x \in\left(M_{1}\right)_{\mathbb{R}} \times\left(M_{2}\right)_{\mathbb{R}}$ is as follows:

$$
x=\sum_{i=1}^{r} a_{i} e_{i}+\sum_{i=j}^{s} b_{j} f_{j}
$$

where $a_{i}, b_{j} \geq 0$ and $\sum a_{i} \leq \sum b_{j} \leq 1$.

The union of the unimodular simplexes contained in $\Delta_{1} \times \Delta_{2}$ is a closed subset. Therefore if $x$ is not contained in any modular subsimplex of $\Delta_{1} \times \Delta_{2}$ then neither is any small perturbation of $x$. Thus we are free to assume that all $a_{i}$ 's and $b_{j}$ 's are nonzero and any two non-empty subsets of $a_{i}$ 's and $b_{j}$ 's have different sum, in particular $a_{1}+\cdots+a_{p} \neq b_{1}+\cdots+b_{q}$ for any reasonable $(p, q)$. Let $m$ be such $b_{1}+\cdots+b_{m-1}<$ $a_{1}+\cdots+a_{r}<b_{1}+\cdots+b_{m}$. We set $b_{m}^{\prime}=\left(b_{1}+\cdots+b_{m}\right)-\left(a_{1}+\cdots+a_{r}\right)$.

In order to prove the lemma we will find $r+m-1$ positive numbers $c_{i, j}$ indexed by some pairs $(i, j) \in\{1, \ldots, r\} \times\{1, \ldots, m\}$ such that

$$
x=\sum_{(i, j)} c_{i, j}\left(e_{i}+f_{j}\right)+b_{m}^{\prime} f_{m}+b_{m+1} f_{m}+\cdots+b_{s} f_{s}
$$

where the sum is over the chosen pairs (for the other pairs $(i, j)$ the coefficients $c_{i, j}$ are assumed to be zero) and the set of corresponding vectors $e_{i}+f_{j}$ together with $f_{m}, \ldots, f_{s}$ can be modified via addition and subtraction to the standard basis $e_{1}, \ldots, e_{r}, f_{1}, \ldots, f_{s}$.

The coefficients $c_{i, j}$ are defined inductively according to the following rules. The first coefficient is $c_{1,1}=\min \left\{a_{1}, b_{1}\right\}$. Suppose that the last coefficient defined is $c_{i_{0}, j_{0}}$. If $\left(i_{0}, j_{0}\right)=(r, m)$ then we are done so assume that it is not the case. Then, because of our assumption that the sequences $\left(a_{i}\right)$ and $\left(b_{j}\right)$ have no equal partial sums, either $a_{1}+\cdots+a_{i_{0}}>b_{1}+\cdots+b_{j_{0}}$, or $a_{1}+\cdots+a_{i_{0}}<b_{1}+\cdots+b_{j_{0}}$. In the former case we set

$$
c_{i_{0}, j_{0}+1}=\min \left\{b_{j_{0}+1},\left(a_{1}+\cdots+a_{i_{0}}\right)-\left(b_{1}+\cdots+b_{j_{0}}\right)\right\},
$$

whereas in the latter case we define

$$
c_{i_{0}+1, j_{0}}=\min \left\{a_{i_{0}+1},\left(b_{1}+\cdots+b_{j_{0}}\right)-\left(a_{1}+\cdots+a_{i_{0}}\right)\right\} .
$$

The verification that $\sum_{i=1}^{r} c_{i, j}=b_{j}$ for $j=1, \ldots, m-1, \sum_{i=1}^{r} c_{i, m}=b_{m}-b_{m}^{\prime}$ and $\sum_{j=1}^{m} c_{i, j}=a_{i}$ for $i=1, \ldots, r$ is left to the reader. Also, a simple backtracking allows us to modify the set consisting of the corresponding vectors $e_{i}+f_{j}$ and $f_{m}, \ldots, f_{s}$ to the standard basis for $M_{1} \times M_{2}$. 
Lemma A.3. Let $\Delta_{1} \subset\left(M_{1}\right)_{\mathbb{R}}$ and $\Delta_{2} \subset\left(M_{2}\right)_{\mathbb{R}}$ be two unimodular simplexes. Consider two homomorphisms $\ell_{i}: M_{i} \rightarrow \mathbb{Z}$ such that $\left(\ell_{i}\right)_{\mathbb{R}}\left(\Delta_{i}\right) \subset[0,1]$. Then the fiber product $\Delta=\left(\Delta_{1}\right) \ell_{1} \times \ell_{2}\left(\Delta_{2}\right)$ has a unimodular covering with respect to the fiber product lattice $M=M_{1} \ell_{1} \times \ell_{2} M_{2}$.

Proof. The argument is a variation of the one used in the previous lemma. We can assume that $\Delta_{1}$ has vertices $0, e_{1}^{0}, \ldots, e_{r_{0}}^{0}, e_{1}^{1}, \ldots, e_{r_{1}}^{1}$ and $\Delta_{2}$ has vertices $0, f_{1}^{0}, \ldots, f_{s_{0}}^{0}$, $f_{1}^{1}, \ldots, f_{s_{1}}^{1}$ where $\ell_{1}\left(e_{i}^{0}\right)=\ell_{2}\left(f_{j}^{0}\right)=0$ and $\ell_{1}\left(e_{i}^{1}\right)=\ell_{2}\left(f_{j}^{1}\right)=1$ for suitable $i$ 's and $j$ 's. Suppose that $x \in\left(M_{1}\right)_{\mathbb{R}} \times\left(M_{2}\right)_{\mathbb{R}}$ is as follows:

$$
x=\sum_{i=1}^{r_{0}} a_{i}^{0} e_{i}^{0}+\sum_{i=1}^{r_{1}} a_{i}^{1} e_{i}^{1}+\sum_{j=1}^{s_{0}} b_{j}^{0} f_{j}^{0}+\sum_{j=1}^{s_{1}} b_{j}^{1} f_{j}^{1}
$$

where $a_{i}^{0}, a_{i}^{1}, b_{j}^{0}, b_{j}^{1} \geq 0, \sum a_{i}^{0}+\sum a_{i}^{1} \leq 1, \sum b_{j}^{0}+\sum b_{j}^{1} \leq 1$ and moreover $\sum a_{i}^{1}=$ $\sum b_{j}^{1}$. The last condition ensures that $\ell_{1}(x)=\ell_{2}(x)$ and it is the only condition which cannot be perturbed as in the proof of the previous lemma.

We write $x=x_{0}+x_{1}$ where $x_{0}=\sum a_{i}^{0} e_{i}^{0}+\sum b_{j}^{0} f_{j}^{0}$ and $x_{1}=\sum a_{i}^{1} e_{i}^{1}+\sum b_{j}^{1} f_{j}^{1}$ and we repeat the proof of A.2 for $x_{0}$ and $x_{1}$ separately. The only difference is that, because of the equality $\sum a_{i}^{1}=\sum b_{j}^{1}$, the construction will give $r_{1}+s_{1}-1$ coefficients $c_{i, j}^{1}$ and associated pairs of vectors $e_{i}^{1}+f_{j}^{1}$, which will enable us to write $x_{1}=\sum c_{i, j}^{1}\left(e_{i}^{1}+f_{j}^{1}\right)$. Thus, clearly, the vectors $e_{i}^{1}+f_{j}^{1}$ do not constitute a basis of the lattice spanned by $e_{1}^{1}, \ldots, e_{r_{1}}^{1}, f_{1}^{1}, \ldots, f_{s_{1}}^{1}$ but of this lattice intersected with $\operatorname{ker}\left(\ell_{1}-\ell_{2}\right)$. That is, among the chosen $r_{1}+s_{1}-1$ vectors $e_{i}^{1}+f_{j}^{1}$ we have $e_{1}^{1}+f_{1}^{1}$ and $e_{r_{1}}^{1}+f_{s_{1}}^{1}$, and if $e_{i}^{1}+f_{j}^{1}$ is chosen then so is either $e_{i+1}^{1}+f_{j}^{1}$ or $e_{i}^{1}+f_{j+1}^{1}$ (but not both). We have to prove that any vector $e_{i}^{1}+f_{j}^{1}$, where $i=1, \ldots, r_{1}, j=1, \ldots, s_{1}$, can be obtained as an integral coefficient sum of the $r_{1}+s_{1}-1$ vectors chosen in our algorithm. But this follows because

$$
\left(e_{i}^{1}+f_{j}^{1}\right)+\left(e_{i+1}^{1}+f_{j+1}^{1}\right)=\left(e_{i+1}^{1}+f_{j}^{1}\right)+\left(e_{i}^{1}+f_{j+1}^{1}\right)
$$

so any one of the above four vectors is a combination of the other three and this observation can be used repeatedly to prove our claim.

Corollary A.4. Let $\Delta_{1} \subset\left(M_{1}\right)_{\mathbb{R}}$ and $\Delta_{2} \subset\left(M_{2}\right)_{\mathbb{R}}$ be two polytopes which have unimodular coverings. Consider two homomorphisms $\ell_{i}: M_{i} \rightarrow \mathbb{Z}$ such that $\left(\ell_{i}\right)_{\mathbb{R}}\left(\Delta_{i}\right) \subset$ $[0,1]$. Then the fiber product $\Delta=\Delta_{1} \ell_{1} \times \ell_{2} \Delta_{2}$ has a unimodular covering with respect to the fiber product lattice $M=M_{1} \ell_{1} \times \ell_{2} M_{2}$.

Proof. The fiber product of $\Delta_{1}$ and $\Delta_{2}$ is covered by fiber products of simplexes from the unimodular covers of each of them. Thus the result follows by A.3

Since the polytope of the star 3-valent tree is a unit tetrahedron, because of 1.13 by induction on the number of inner nodes we get the following result.

Proposition A.5. If $\mathcal{T}$ is a trivalent tree then its polytope $\Delta(\mathcal{T})$ in $\widehat{M}(\mathcal{T})$ has a unimodular covering, hence it is normal. 


\section{A.2. Two 3-valent trees with six leaves}

One of the fundamental questions regarding phylogenetic trees is the following. Given two trees $\mathcal{T}_{1}$ and $\mathcal{T}_{2}$ suppose that $\Delta\left(\mathcal{T}_{1}\right) \cong \Delta\left(\mathcal{T}_{2}\right)$ as lattice polytopes, or the projective models $X\left(\mathcal{T}_{1}\right)$ and $X\left(\mathcal{T}_{2}\right)$ are projectively equivalent. Does this imply that the trees are equivalent (as $\mathrm{CW}$ complexes) as well?

We tackled this problem and compared models of the two simplest non-equivalent trees. These are the 6-leaf trees pictured below, a 3-caterpillar and a snow flake tree [StSu].
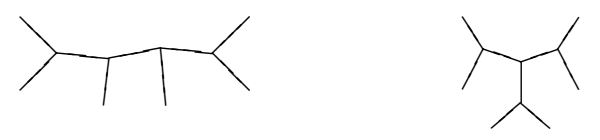

The snow flake tree is obtained from the 3-caterpillar tree by elementary mutation along its middle inner edge. Therefore their Hilbert-Ehrhart polynomials are equal and computed with [maxima] to be as follows:

$$
\begin{aligned}
h(n)= & \frac{1}{22680}(n+1)(n+2)(n+3) \\
& \cdot\left(31 n^{6}+372 n^{5}+1942 n^{4}+5616 n^{3}+9511 n^{2}+8988 n+3780\right) .
\end{aligned}
$$

On the other hand, we can distinguish their polytopes in terms of some combinatorial invariants.

Given a polytope $\Delta$ we define its incidence matrix $\left(a_{i j}\right)$ as follows: $\left(a_{i j}\right)$ is a symmetric matrix with integral entries such that for $i \leq j$ the number $a_{i j}$ is equal to the number of $i$-dimensional faces contained in $j$-dimensional faces of $\Delta$. In particular $a_{i i}$ is the number of $i$-dimensional faces. The following is the incidence matrix of the polytope of the snow flake tree:

$\begin{array}{ccccccccc}32 & 480 & 2400 & 6144 & 9312 & 8832 & 5280 & 1920 & 384 \\ 480 & 240 & 2400 & 9456 & \mathbf{1 9 9 2 0} & \mathbf{2 4 9 6 0} & \mathbf{1 9 2 0 0} & \mathbf{8 8 8 0} & \mathbf{2 2 5 6} \\ 2400 & 2400 & 760 & 5944 & \mathbf{1 9 0 0 8} & \mathbf{3 2 5 5 2} & \mathbf{3 2 4 0 8} & \mathbf{1 8 7 9 2} & \mathbf{5 8 7 2} \\ 6144 & 9456 & 5944 & 1316 & \mathbf{8 4 0 0} & \mathbf{2 1 7 4 4} & \mathbf{2 9 3 0 8} & \mathbf{2 1 7 2 0} & \mathbf{8 3 8 8} \\ 9312 & \mathbf{1 9 9 2 0} & \mathbf{1 9 0 0 8} & \mathbf{8 4 0 0} & 1392 & \mathbf{7 2 0 0} & \mathbf{1 4 6 4 0} & \mathbf{1 4 6 4 0} & \mathbf{7 2 0 0} \\ 8832 & \mathbf{2 4 9 6 0} & \mathbf{3 2 5 5 2} & \mathbf{2 1 7 4 4} & \mathbf{7 2 0 0} & 940 & \mathbf{3 8 2 0} & \mathbf{5 7 6 0} & \mathbf{3 8 2 0} \\ 5280 & \mathbf{1 9 2 0 0} & \mathbf{3 2 4 0 8} & \mathbf{2 9 3 0 8} & \mathbf{1 4 6 4 0} & \mathbf{3 8 2 0} & 406 & 1224 & 1224 \\ 1920 & \mathbf{8 8 8 0} & \mathbf{1 8 7 9 2} & \mathbf{2 1 7 2 0} & \mathbf{1 4 6 4 0} & \mathbf{5 7 6 0} & 1224 & 108 & 216 \\ 384 & \mathbf{2 2 5 6} & \mathbf{5 8 7 2} & \mathbf{8 3 8 8} & \mathbf{7 2 0 0} & \mathbf{3 8 2 0} & 1224 & 216 & 16\end{array}$

And this is the incidence matrix of the polytope of the 3-caterpillar tree:

$\begin{array}{ccccccccc}32 & 480 & 2400 & 6144 & 9312 & 8832 & 5280 & 1920 & 384 \\ 480 & 240 & 2400 & 9456 & \mathbf{1 9 9 0 4} & \mathbf{2 4 8 9 6} & \mathbf{1 9 1 0 4} & \mathbf{8 8 1 6} & \mathbf{2 2 4 0} \\ 2400 & 2400 & 760 & 5944 & \mathbf{1 8 9 7 6} & \mathbf{3 2 4 0 8} & \mathbf{3 2 1 6 8} & \mathbf{1 8 6 1 6} & \mathbf{5 8 2 4} \\ 6144 & 9456 & 5944 & 1316 & \mathbf{8 3 8 4} & \mathbf{2 1 6 4 8} & \mathbf{2 9 1 1 2} & \mathbf{2 1 5 5 2} & \mathbf{8 3 3 6} \\ 9312 & \mathbf{1 9 9 0 4} & \mathbf{1 8 9 7 6} & \mathbf{8 3 8 4} & 1392 & \mathbf{7 1 8 4} & \mathbf{1 4 5 8 4} & \mathbf{1 4 5 7 6} & \mathbf{7 1 7 6} \\ 8832 & \mathbf{2 4 8 9 6} & \mathbf{3 2 4 0 8} & \mathbf{2 1 6 4 8} & \mathbf{7 1 8 4} & 940 & \mathbf{3 8 1 6} & \mathbf{5 7 5 2} & \mathbf{3 8 1 6} \\ 5280 & \mathbf{1 9 1 0 4} & \mathbf{3 2 1 6 8} & \mathbf{2 9 1 1 2} & \mathbf{1 4 5 8 4} & \mathbf{3 8 1 6} & 406 & 1224 & 1224 \\ 1920 & \mathbf{8 8 1 6} & \mathbf{1 8 6 1 6} & \mathbf{2 1 5 5 2} & \mathbf{1 4 5 7 6} & \mathbf{5 7 5 2} & 1224 & 108 & 216 \\ 384 & \mathbf{2 2 4 0} & \mathbf{5 8 2 4} & \mathbf{8 3 3 6} & \mathbf{7 1 7 6} & \mathbf{3 8 1 6} & 1224 & 216 & 16\end{array}$


Both matrices were computed by [polymake]. We note that although both polytopes have the same number of faces of the same dimension their incidences are different (indicated in boldface).

Acknowledgments. We would like to thank Jarosław Buczyński for his remarks, Piotr Zwiernik for bringing this subject to our attention and the referee for remarks which greatly improved the exposition.

Research of J. A. Wiśniewski was partially supported by Polish KBN (grant 1P03A03027) and MPIM, Bonn, Germany.

\section{References}

[AlRh] Allman, E. S., A. Rhodes, J.: The identifiability of tree topology for phylogenetic models, including covarion and mixture models. J. Comput. Biol. 13, 1101-1113 (2006) MR 2197150

[Alt] Altmann, K.: Deformation Theory. Notes available at http://page.mi.fu-berlin.de/ altmann/PAPER/dmv.ps (1995)

[B-B] Białynicki-Birula, A.: Quotients by actions of groups. In: Encyclopedia Math. Sci. 131, Springer, 1-82 (2003) Zbl 1061.14046 MR 1925829

[BHV] Billera, L., Holmes, S. P., Vogtmann, K.: Geometry of the space of phylogenetic trees. Adv. Appl. Math. 27, 733-767 (2001) Zbl 0995.92035 MR 1867931

[BGT] Bruns, W., Gubeladze, J., Trung, N. V.: Normal polytopes, triangulations and Koszul algebras. J. Reine Angew. Math. 485, 123-160 (1997) Zbl 0866.20050 MR 1442191

[BuWi] Buczyńska, W., Wiśniewski, J. A.: On phylogenetic trees-a geometer's view. math.AG/0601357

[CGS] Casanellas, M., Garcia, L. D., Sullivant, S.: Catalog of small trees. In: Algebraic Statistics for Computational Biology, L. Pachter and B. Sturmfels (eds.), Cambridge Univ. Press, 291-304 (2005)

[Ei] Eisenbud, D.: Commutative Algebra with a View Toward Algebraic Geometry. Springer (1995). Zbl 0819.13001 MR 1322960

[EiSt] Eisenbud, D., Sturmfels, B.: Binomial ideals. Duke Math. J. 84, 1-45 (1996) Zbl 0873.13021 MR 1394747

[ERSS] Eriksson, N., Ranestad, K., Sturmfels, B., Sullivant, S.: Phylogenetic algebraic geometry. In: Projective Varieties with Unexpected Properties, de Gruyter, 237-255 (2005) Zbl pre05054808 MR 2202256

[EvSp] Evans, S., Speed, T.: Invariants of some probability models used in phylogenetic inference. Ann. Statist. 21, 355-377 (1993) Zbl 0772.92012 MR 1212181

[Fe] Felsenstein, J.: Inferring Phylogenies. Sinauer Associates (2003)

[Fu] Fulton, W.: Introduction to Toric Varieties. Princeton Univ. Press (1993) Zbl 0813.14039 MR 1234037

[Ha] Hartshorne, R.: Algebraic Geometry. Grad. Texts in Math. 52, Springer (1977) Zbl 0367.14001 MR 0463157

[KoMo] Kollár, J., Mori, Sh.: Birational Geometry of Algebraic Varieties. Cambridge Univ. Press (1998) Zbl 0926.14003 MR 1658959

[Mu Mumford, D., Fogarty, J.: Geometric Invariant Theory. 2nd ed., Springer (1982) Zbl 0504.14008 MR 0719371

[Na] Namikawa, Y.: Smoothing Fano 3-folds. J. Algebraic Geom. 6, 307-324 (1997) Zbl 0906.14019 MR 1489117 
[Oda] Oda, T.: Convex Bodies and Algebraic Geometry. Springer (1988) Zbl 0628.52002 MR 0922894

[PaSt] Pachter, L., Sturmfels, B. (eds.): Algebraic Statistics for Computational Biology. Cambridge Univ. Press (2005) Zbl pre02233465 MR 2205869

[Re] Reid, M.: What is a flip?. Colloquium talk notes, Salt Lake City (1992), http://www.maths.warwick.ac.uk $/$ miles/3folds/what_flip.pdf

[SeSt] Semple, C., Steel, M.: Phylogenetics. Oxford Univ. Press (2003) Zbl 1043.92026 MR 2060009

[St1] Sturmfels, B.: Equations defining toric varieties. In: Algebraic Geometry (Santa Cruz, 1995), Proc. Sympos. Pure Math. 62, Amer. Math. Soc., 437-449 (1997). Zbl 0914.14022 MR 1492542

[St2] Sturmfels, B.: Gröbner Bases and Convex Polytopes. Univ. Lecture Ser. 8, Amer. Math. Soc. (1996) Zbl 0856.13020 MR 1363949

[StSu] Sturmfels, B., Sullivant, S.: Toric ideals of phylogenetic invariants. J. Comput. Biol. 12, 204-228 (2005)

[SSE] Szekely, L., Steel, M., Erdős, P.: Fourier calculus on evolutionary trees. Adv. Appl. Math. 14, 200-216 (1993) Zbl 0794.05014 MR 1218244

[maxima ] Maxima 5.4, W. Schelter et al., http://maxima.sourceforge.net/

[polymake] Polymake 2.0.1, E. Gavrilow, M. Joswig et al., http://www.math.tu-berlin.de/ polymake 